\title{
THE FUNDAMENTAL LEPAGE FORM IN VARIATIONAL THEORY FOR SUBMANIFOLDS
}

\author{
ZBYNĚK URBAN AND JÁN BRAJERČÍK
}

\begin{abstract}
A setting for global variational geometry on Grassmann fibrations is presented. The integral variational functionals for finite dimensional immersed submanifolds are studied by means of the fundamental Lepage equivalent of a homogeneous Lagrangian, which can be regarded as a generalization of the well-known Hilbert form in the classical mechanics. Prolongations of immersions, diffeomorphisms and vector fields to the Grassmann fibrations are introduced as geometric tools for the variations of immersions. The first infinitesimal variation formula together with its consequences, the Euler-Lagrange equations for extremal submanifolds and the Noether theorem for invariant variational functionals are proved. The theory is illustrated on the variational functional for minimal submanifolds.
\end{abstract}

\section{INTRODUCTION}

The subject of this paper is the theory of variational functionals for submanifolds, where extremals become submanifolds of Euclidean spaces or general finite dimensional smooth manifolds. Thus, the variational variables will be rather sets than mappings between manifolds. This theory requires adequate underlying geometric structures, the quotient topological spaces the higher-order Grassmann fibrations. Following the pioneering work by Dedecker [6], the theory has been studied by different authors (cf. Crampin and Saunders [4, Manno and Vitolo [18, Grigore [9]), who employed different geometric structures and variational objects defined on them. The variational theory for one-dimensional immersed submanifolds (fibered mechanics), including the Noether-type invariance theory, was also studied by Urban and Krupka [21, 22, 23] with a direct use of Grassmann fibrations as the underlying spaces.

Our main aim is to develop foundations of multiple-integral variational functionals for submanifolds, defined by differential forms with specific properties - Lepage forms. Roughly speaking, Lepage forms represent a far-going generalization of the well-known Cartan form from the calculus of variations of simple integral problems and classical mechanics. Replacing the initial Lagrangian by its Lepage equivalent, one obtains the same variational functional, but additionaly the geometric and variational properties of the functional are described by geometric operations acting on the corresponding Lepage equivalent. For a review of basic properties and results

2000 Mathematics Subject Classification. 58E30; 58A20; 58D19; 53A10.

Key words and phrases. Lagrangian; Euler-Lagrange form; Lepage equivalent; Noether current; Grassmann fibration; Zermelo conditions; minimal surface functional.

The authors are very grateful to Professors Demeter Krupka and David J. Saunders for research discussions during the 22nd International Summer School on Global Analysis and Applications, Krakow, August 2017. ZU also acknowledges the SAIA scholarship programme, and hospitality of the Department of Physics, Mathematics and Techniques, University of Prešov. 
of the theory of Lepage forms in the calculus of variations, see Krupka, Krupková, and Saunders [17].

We introduce the concept of a Lepage form for differential forms on manifolds of regular velocities (jets of immersions) with the help of the canonical embedding into fibered velocity spaces. It appears that these forms can be canonically projected onto the Grassmann fibrations, hence we obtain crucial objects for studying variational properties (namely variations, extremals, and invariance properties) of the integral functionals. To this purpose we utilize the concept of the fundamental Lepage equivalent of a Lagrangian, introduced by Krupka [12] on first-order jet prolongation of a fibered manifold over an $n$-dimensional base. This particular Lepage equivalent is also characterized by the following important property: it is closed if and only if the corresponding Lagrangian is trivial (i.e. the Euler-Lagrange expressions vanish identically). This fact can also be profitably applied in formulation of first-order local variational principles (cf. Brajerčík and Krupka 2]). In such situation, a global Lagrangian for the variational functional need not be defined. The problem how to reconstruct the variational functional from the local data occurs in many physical theories (see Giachetta, Mangiarotti, and Sardanashvily [8]).

In Section 2, we briefly summarize basic concepts of Lepage forms in first-order variational field theory on fibered manifolds, including a description of the wellknown examples of Lepage equivalents, namely the Poincaré-Cartan form, the fundamental Lepage form, and the Carathéodory form (cf. Carathéodory [3]). Section 3 contains the geometric structure of manifolds of velocities and Grassmann fibrations, adapted to our setting. A particular attention is devoted to the Grassmann prolongations of diffeomorphisms and vector fields, used later on within the calculus of variations. In Section 4, we study the fundamental Lepage equivalent of a positive-homogeneous Lagrangian, in particular, we derive its local structure and prove that this differential $n$-form is defined on the Grassmann fibration. Necessary and sufficient conditions for a function on manifold of regular velocities to be positive homogeneous (the Zermelo conditions, see e.g. McKiernan [19], Urban and Krupka [24]) are applied. In this sense, we follow the idea of Krupka [15], who studied the unique Lepage equivalent of an $r$-th order Lagrangian in fibered mechanics (the generalization of the Cartan form) under assumption of a positivehomogeneous Lagrangian hence obtaining a generalization of the Hilbert form. An alternative approach was applied by Crampin and Saunders [4, whose starting object is the Carathéodory form in first-order field theory for a positive-homogeneous Lagrangian, resulting into the Hilbert-Carathéodory form, which differs from the fundamental Lepage equivalent (see Remark 18). These two Lepage equivalents are, however, very closely related as they both define the minimal submanifold problems (Section 6).

In Section 5, the first-order variational field theory for submanifolds is developed. First we study conditions under which a differential $n$-form on the manifold of regular $n$-velocities is a Lepage form (Theorem 19), and observe that horizontal component of a Lepage form is given by a positive-homogeneous function. This allows us to employ the fundamental Lepage equivalent of a homogeneous Lagrangian as a basic element of the theory. We derive the infinitesimal first variation formula and its consequences for extremals and conservation laws in a global sense. Furthermore, we extend the classical invariant variational principles and the Noether 
theory (see e.g. Kossmann-Schwarzbach [11, Krupka [16]) to functionals given by Lepage forms on the Grassmann fibrations.

The results and methods of this work are illustrated on classical example of the variational functional for minimal submanifolds (Section 6). In particular, we show that the fundamental Lepage equivalent and the Hilbert-Carathéodory equivalent of the minimal submanifold Lagrangian coincide. Then we analyze the invariance properties of the variational problem of minimal surfaces $(n=2)$. It turns out in this example that the "conservation law" equations are completely equivalent with the Euler-Lagrange equations for extremals.

Basic underlying structures, well adapted to this paper, can be found in Grigore and Krupka [10]. Throughout, we use the standard geometric concepts: the exterior derivative $d$, the contraction $i_{\Xi} \rho$ and the Lie derivative $\partial_{\Xi} \rho$ of a differential form $\rho$ with respect to a vector field $\Xi$, and the pull-back operation $*$ acting on differential forms. If $(U, \varphi), \varphi=\left(x^{j}\right)$, is a chart on smooth manifold $X$, we set

$$
\begin{aligned}
& \omega_{0}=\frac{1}{n !} \varepsilon_{j_{1} j_{2} \ldots j_{n}} d x^{j_{1}} \wedge d x^{j_{2}} \wedge \ldots \wedge d x^{j_{n}}, \\
& \omega_{j}=i_{\partial / \partial x^{j}} \omega_{0}=\frac{1}{(n-1) !} \varepsilon_{j i_{2} \ldots i_{n}} d x^{i_{2}} \wedge \ldots \wedge d x^{i_{n}},
\end{aligned}
$$

where $\varepsilon_{i_{1} i_{2} \ldots i_{n}}$ is the Levi-Civita permutation symbol.

\section{LEPAGE FORMS IN FIRST-ORDER FIELD THEORY ON FIBERED SPACES}

In this section we summarize basic ideas and results of first-order Lepage forms in the global calculus of variations on fibered spaces over $n$-dimensional manifolds. A comprehensive higher-order treatment can be found in Krupka [16], and in particular first-order field theory was studied by Volná and Urban 25.

Throughout, we denote by $Y$ a fibered manifold of dimension $n+M$ over an $n$-dimensional base $X$ with projection $\pi: Y \rightarrow X$ the surjective submersion. $J^{1} Y$ (resp. $J^{2} Y$ ) denotes the first (resp. second) jet prolongation of $Y$ whose elements are jets $J_{x}^{1} \gamma$ (resp. $J_{x}^{2} \gamma$ ) of sections $\gamma$ of $\pi$ with source $x \in X$ and target $\gamma(x) \in Y$. The canonical jet projections $\pi^{1}: J^{1} Y \rightarrow X$ and $\pi^{1,0}: J^{1} Y \rightarrow Y$ (resp. $\pi^{2}: J^{2} Y \rightarrow X, \pi^{2,0}: J^{2} Y \rightarrow Y, \pi^{2,1}: J^{2} Y \rightarrow J^{1} Y$ ), are defined by $\pi^{1}\left(J_{x}^{1} \gamma\right)=x, \pi^{1,0}\left(J_{x}^{1} \gamma\right)=\gamma(x)$ (resp. $\pi^{2}\left(J_{x}^{2} \gamma\right)=x, \pi^{2,0}\left(J_{x}^{2} \gamma\right)=\gamma(x)$, $\left.\pi^{2,1}\left(J_{x}^{2} \gamma\right)=J_{x}^{1} \gamma\right)$. The jet prolongation $J^{1} \gamma$ of a section $\gamma\left(\right.$ resp. $\left.J^{2} \gamma\right)$, defined on an open subset of $X$, is given by $J^{1} \gamma(x)=J_{x}^{1} \gamma\left(\right.$ resp. $\left.J^{2} \gamma(x)=J_{x}^{2} \gamma\right)$. For an open subset $W$ of $Y$ we put $W^{1}=\left(\pi^{1,0}\right)^{-1}(W), W^{2}=\left(\pi^{2,0}\right)^{-1}(W)$. Let $(V, \psi)$, $\psi=\left(x^{j}, y^{K}\right)$, be a fibered chart on $Y$, and denote by $\left(V^{1}, \psi^{1}\right), \psi^{1}=\left(x^{j}, y^{K}, y_{l}^{K}\right)$ (resp. $\left.\left(V^{2}, \psi^{2}\right), \psi^{2}=\left(x^{j}, y^{K}, y_{l}^{K}, y_{l k}^{K}\right)\right)$, the associated fibered chart on $J^{1} Y$ (resp. $J^{2} Y$ ), and by $(\pi(V), \varphi), \varphi=\left(x^{j}\right)$, the associated chart on $X$, where $y_{l}^{K}\left(J_{x}^{1} \gamma\right)=D_{l}\left(y^{K} \gamma \varphi^{-1}\right)(\varphi(x)), y_{l k}^{K}\left(J_{x}^{2} \gamma\right)=D_{l} D_{k}\left(y^{K} \gamma \varphi^{-1}\right)(\varphi(x))$, and $1 \leq j \leq n$, $1 \leq K \leq M, 1 \leq l \leq k \leq n$. A tangent vector $\xi$ at a point $y \in Y$ is said to be $\pi$ vertical, if $T \pi \cdot \xi=0$, and a differential form $\rho$ on $Y$ is said to be $\pi$-horizontal, if for every point $y \in Y$ the contraction $i_{\xi} \rho(y)$ vanishes whenever $\xi \in T_{y} Y$ is a $\pi$-vertical vector. The concepts of $\pi^{1}-, \pi^{2}-, \pi^{1,0_{-}}$, and $\pi^{2,0}$-horizontal forms are introduced analogously. A vector field $\Xi$ on $Y$ is called $\pi$-projectable, if there exists a vector field $\xi$ on $X$ such that $T \pi \cdot \Xi=\xi \circ \pi$. In a fibered chart $(V, \psi), \psi=\left(x^{j}, y^{K}\right)$, a $\pi$ projectable vector field $\Xi$ has an expression $\Xi=\xi^{j}\left(\partial / \partial x^{j}\right)+\Xi^{K}\left(\partial / \partial y^{K}\right)$, where $\xi^{j}=\xi^{j}\left(x^{l}\right), \Xi^{K}=\Xi^{K}\left(x^{l}, y_{k}^{L}\right)$. 
Let $q \geq 1$ be an integer. For any open set $W \subset Y$, we denote by $\Omega_{q}^{1} W$ (resp. $\Omega_{q}^{2} W$ ) the $\Omega_{0}^{1} W$-module (resp. $\Omega_{0}^{2} W$-module) of smooth differential $q$-forms defined on $W^{1}$ (resp. $W^{2}$ ), where $\Omega_{0}^{1} W$ (resp. $\Omega_{0}^{2} W$ ) is the ring of smooth functions on $W^{1}$ (resp. $W^{2}$ ). Clearly, $\pi^{1}$-horizontal (resp. $\pi^{1,0}$-horizontal) $q$-forms on $W^{1}$ constitute submodule of the $\Omega_{0}^{1} W$-module $\Omega_{q}^{1} W$, denoted by $\Omega_{q, X}^{1} W\left(\right.$ resp. $\left.\Omega_{q, Y}^{1} W\right)$; the modules of $\pi^{2}$-horizontal (resp. $\pi^{2,0}$-horizontal) $q$-forms on $W^{2}$ are denoted by $\Omega_{q, X}^{2} W$ (resp. $\Omega_{q, Y}^{2} W$ ). A morphism of exterior algebras $\Omega_{q}^{1} W \ni \rho \rightarrow h \rho \in \Omega_{q, X}^{2} W$, defined with respect to any fibered chart $(V, \psi), \psi=\left(x^{i}, y^{K}\right)$, by the identities,

$$
h f=f \circ \pi^{2,1}, \quad h d x^{i}=d x^{i}, \quad h d y^{K}=y_{k}^{K} d x^{k}, \quad h d y_{j}^{K}=y_{j k}^{K} d x^{k},
$$

where $f: V^{1} \rightarrow \mathbf{R}$ is a differentiable function, is called the $\pi$-horizontalization. In particular, $h d f=\left(d_{i} f\right) d x^{i}$, where $d_{i} f=\partial f / \partial x^{i}+\left(\partial f / \partial y^{K}\right) y_{i}^{K}+\left(\partial f / \partial y_{j}^{K}\right) y_{j i}^{K}$ is the $i$-th formal derivative operator associated to $(V, \psi)$. Note that for any section $\gamma$ of $Y$, we have $J^{1} \gamma^{*} \rho=J^{2} \gamma^{*} h \rho$. A differential $q$-form $\rho \in \Omega_{q}^{1} W$ is said to be contact, if $J^{1} \gamma^{*} \rho=0$ for all sections $\gamma$ of $Y$ defined on an open subset of $X$ with values in $W$; this condition is equivalent to $h \rho=0$. If $(V, \psi), \psi=\left(x^{i}, y^{K}\right)$, is a fibered chart on $Y$, then the forms $d x^{i}, \omega^{K}, d y_{j}^{K}$, where

$$
\omega^{K}=d y^{K}-y_{l}^{K} d x^{l},
$$

constitute a basis of linear forms on $V^{1}$. For $2 \leq q \leq n$, any contact $q$-form on $W^{1}$ is locally generated by the contact forms $\omega^{K}$ and $d \omega^{K}$ (cf. Krupka [16]). Any differential $q$-form $\rho \in \Omega_{q}^{1} W$ has a unique invariant decomposition

$$
\left(\pi^{2,1}\right)^{*} \rho=h \rho+\sum_{k=1}^{q} p_{k} \rho
$$

where $p_{k} \rho$ is the $k$-contact component of $\rho$, containing exactly $k$ exterior factors $\omega^{K}$ in any fibered chart $(V, \psi)$.

Any element $\lambda \in \Omega_{n, X}^{1} W$, i.e. a $\pi^{1}$-horizontal $n$-form on the open set $W^{1} \subset J^{1} Y$, is called a Lagrangian for $Y$ of order 1 . In a fibered chart $(V, \psi), \psi=\left(x^{i}, y^{K}\right)$, where $V \subset W, \lambda$ is expressed by

$$
\lambda=\mathscr{L} \omega_{0}
$$

where $\omega_{0}=d x^{1} \wedge d x^{2} \wedge \ldots \wedge d x^{n}$ is the (local) volume element and $\mathscr{L}: V^{1} \rightarrow \mathbf{R}$ is the Lagrange function, associated to $\lambda$.

In accordance with the general theory of Lepage forms (cf. Krupka [13]), we say that an $n$-form $\Theta_{\lambda} \in \Omega_{n}^{1} W$ is a Lepage equivalent of $\lambda$ on $W^{1} \subset J^{1} Y$, if the following two conditions are satisfied:

(a) $h \Theta_{\lambda}=\left(\pi^{2,1}\right)^{*} \lambda$ (i.e. $\Theta_{\lambda}$ is equivalent with $\lambda$ ), and

(b) $h i_{\xi} d \Theta_{\lambda}=0$ for arbitrary $\pi^{1,0}$-vertical vector field $\xi$ on $W^{1}$ (i.e. $\Theta_{\lambda}$ is a Lepage form).

The following two theorems describe the structure of Lepage forms and Lepage equivalents of a Lagrangian (see Krupka [13, 16]).

Theorem 1. An $n$-form $\rho \in \Omega_{n}^{1} W$ is a Lepage form if and only if for any fibered chart $(V, \psi), \psi=\left(x^{i}, y^{K}\right)$, on $Y$, where $V \subset W$,

$$
\left(\pi^{2,1}\right)^{*} \rho=\Theta+d \mu+\eta,
$$


where the principal component $\Theta$ is expressed as

$$
\Theta=f_{0} \omega_{0}+\left(\frac{\partial f_{0}}{\partial y_{j}^{K}}-d_{p} \frac{\partial f_{0}}{\partial y_{p j}^{K}}\right) \omega^{K} \wedge \omega_{j}+\frac{\partial f_{0}}{\partial y_{i j}^{K}} \omega_{i}^{K} \wedge \omega_{j},
$$

$f_{0}$ is a differentiable function on $V^{2} \subset J^{2} Y$, given by $h \rho=f_{0} \omega_{0}, \mu$ is a contact $(n-1)$-form, and an $n$-form $\eta$ has the order of contactness $\geq 2$.

Corollary 2. Let $\lambda \in \Omega_{n}^{1} W$ be a Lagrangian of order 1 , expressed by $\lambda=\mathscr{L} \omega_{0}$. A Lepage form $\rho \in \Omega_{n}^{1} W$ is a Lepage equivalent of $\lambda$ if and only if the principal component $\Theta$ of $\rho$ is defined on $W^{1}$ and has an expression

$$
\Theta=\mathscr{L} \omega_{0}+\frac{\partial \mathscr{L}}{\partial y_{j}^{K}} \omega^{K} \wedge \omega_{j} .
$$

Theorem 3. Let $\rho \in \Omega_{n}^{1} W$ be a Lepage form expressed by (2.2). Then

$$
\left(\pi^{2,1}\right)^{*} d \rho=E+F
$$

where $E$ is a 1-contact, $\pi^{2,0}$-horizontal $(n+1)$-form, which has a chart expression

$$
E=\left(\frac{\partial f_{0}}{\partial y^{K}}-d_{j} \frac{\partial f_{0}}{\partial y_{j}^{K}}\right) \omega^{K} \wedge \omega_{0}
$$

with $h \rho=f_{0} \omega_{0}$, and a form $F$ has the order of contactness $\geq 2$.

In the class of Lepage equivalents of any first-order Lagrangian we have a possibility to determine a unique Lepage equivalent by means of additional requirements. Let $\lambda \in \Omega_{n, X}^{1} W$ be a Lagrangian, $\lambda=\mathscr{L} \omega_{0}$ with respect to a fibered chart $(V, \psi), \psi=\left(x^{i}, y^{K}\right)$, on $Y$. The following theorems describe some well-known examples.

Theorem 4. (Poincaré-Cartan equivalent) There exists a unique Lepage equivalent $\Theta_{\lambda} \in \Omega_{n, Y}^{1} W$ of $\lambda$ such that the order of contactness of $\Theta_{\lambda}$ is $\leq 1$. $\Theta_{\lambda}$ has a local expression (2.3) with respect to a fibered chart $(V, \psi)$.

Theorem 5. (Fundamental Lepage equivalent) The differential $n$-form $Z_{\lambda} \in$ $\Omega_{n}^{1} W$, given in a chart $(V, \psi)$ by the expression

$$
\begin{aligned}
Z_{\lambda}= & \mathscr{L} \omega_{0}+\sum_{k=1}^{n} \frac{1}{(n-k) !} \frac{1}{(k !)^{2}} \frac{\partial^{k} \mathscr{L}}{\partial y_{j_{1}}^{K_{1}} \partial y_{j_{2}}^{K_{2}} \ldots \partial y_{j_{k}}^{K_{k}}} \varepsilon_{j_{1} j_{2} \ldots j_{k} i_{k+1} i_{k+2} \ldots i_{n}} \\
& \cdot \omega^{K_{1}} \wedge \omega^{K_{2}} \wedge \ldots \wedge \omega^{K_{k}} \wedge d x^{i_{k+1}} \wedge d x^{i_{k+2}} \wedge \ldots \wedge d x^{i_{n}}
\end{aligned}
$$

is a Lepage equivalent of the first-order Lagrangian $\lambda$.

Remark 6. The crucial property of the fundamental Lepage equivalent is characterized by two equivalent conditions: (i) $Z_{\lambda}$ is closed, (ii) $\lambda$ is trivial (i.e. the Euler-Lagrange expressions associated with $\lambda$ vanish identically). Moreover, if $\rho \in \Omega_{n}^{0} W$, then $Z_{h \rho}=\left(\pi^{1,0}\right)^{*} \rho$. This equivalent was discovered by Krupka [12] (see also Betounes [1]). Attempts to generalize the fundamental Lepage equivalent to higher-order spaces appeared for dimension $n=2$ only, cf. Saunders and Crampin [20]. 
Theorem 7. (Carathéodory equivalent) Let $\lambda \in \Omega_{n, X}^{1} W$ be a non-vanishing first-order Lagrangian. Then the differential $n$-form $\Lambda_{\lambda} \in \Omega_{n}^{1} W$, given in a chart $(V, \psi)$ by the expression

$$
\begin{aligned}
\Lambda_{\lambda}=\mathscr{L} & \left(d x^{1}+\frac{1}{\mathscr{L}} \frac{\partial \mathscr{L}}{\partial y_{1}^{\sigma_{1}}} \omega^{\sigma_{1}}\right) \wedge\left(d x^{2}+\frac{1}{\mathscr{L}} \frac{\partial \mathscr{L}}{\partial y_{2}^{\sigma_{2}}} \omega^{\sigma_{2}}\right) \\
& \wedge \ldots \wedge\left(d x^{n}+\frac{1}{\mathscr{L}} \frac{\partial \mathscr{L}}{\partial y_{n}^{\sigma_{n}}} \omega^{\sigma_{n}}\right),
\end{aligned}
$$

is a Lepage equivalent of $\lambda$.

Remark 8. The $n$-form $\Lambda_{\lambda}$ (2.5) is called the Carathéodory form. It can be shown that $\Lambda_{\lambda}$ is invariant with respect to all coordinate transformations on $Y$ (see Carathéodory [3], Dedecker [6]).

The next lemma is needed for further proofs.

Lemma 9. Suppose a q-form $\rho \in \Omega_{q}^{1} W$ has a chart expression

$$
\rho=\sum_{k=0}^{q} \frac{1}{k !(q-k) !} B_{K_{1} \ldots K_{k} i_{k+1} \ldots i_{q}} \omega^{K_{1}} \wedge \ldots \wedge \omega^{K_{k}} \wedge d x^{i_{k+1}} \wedge \ldots \wedge d x^{i_{q}}
$$

with the coefficients skew-symmetric in all indices $K_{1}, \ldots, K_{k}$, and in all indices $i_{k+1}, \ldots, i_{q}$. Then

$$
\rho=\sum_{k=0}^{q} \frac{1}{k !(q-k) !} A_{K_{1} \ldots K_{k} i_{k+1} \ldots i_{q}} d y^{K_{1}} \wedge \ldots \wedge d y^{K_{k}} \wedge d x^{i_{k+1}} \wedge \ldots \wedge d x^{i_{q}}
$$

where

$$
\begin{gathered}
A_{K_{1} \ldots K_{k} i_{k+1} \ldots i_{q}}=\sum_{l=k}^{q}(-1)^{l-k}\left(\begin{array}{c}
q-k \\
q-l
\end{array}\right) B_{K_{1} \ldots K_{l} i_{l+1} \ldots i_{q}} y_{i_{k+1}}^{K_{k+1}} \ldots y_{i_{l}}^{K_{l}} \\
\quad \operatorname{Alt}\left(i_{k+1}, \ldots, i_{q}\right) .
\end{gathered}
$$

Proof. If a $q$-form $\rho \in \Omega_{q, Y}^{1} W$ has a chart expression (2.6), then the contact components $p_{k} \rho$ of $\rho$, where $0 \leq k \leq q$, are given by

$$
p_{k} \rho=\frac{1}{k !(q-k) !} B_{K_{1} \ldots K_{k} i_{k+1} \ldots i_{q}} \omega^{K_{1}} \wedge \ldots \wedge \omega^{K_{k}} \wedge d x^{i_{k+1}} \wedge \ldots \wedge d x^{i_{q}}
$$

where

$$
B_{K_{1} \ldots K_{k} i_{k+1} \ldots i_{q}}=\sum_{l=k}^{q}\left(\begin{array}{c}
q-k \\
q-l
\end{array}\right) A_{K_{1} \ldots K_{l} i_{l+1} \ldots i_{q}} y_{i_{k+1}}^{K_{k+1}} \ldots y_{i_{l}}^{K_{l}} \operatorname{Alt}\left(i_{k+1}, \ldots, i_{q}\right)
$$

(a proof can be found in Krupka [16] for differential forms on arbitrary finite-order jet prolongation of a fibered manifold). The identities (2.8) for all $k, 0 \leq k \leq q$, constitute, however, a system of multi-linear equations which can be directly solved with respect to the coefficients $A_{K_{1} \ldots K_{k} i_{k+1} \ldots i_{q}}$. To obtain expressions (2.7), one can proceed by induction with respect to degree $k$ of contactness of $\rho$. 


\section{First-ORDER VELOCITIES AND GRASSMANN FIBRATIONS}

From now on, the concepts and results of Section 2 will be employed for the case of a fibered manifold $Y=\mathbf{R}^{n} \times Q$, where $Q$ is a smooth manifold of dimension $M=n+m$ for positive integers $n, m$. The Cartesian coordinates of $\mathbf{R}^{n}$ are denoted by $x^{i}, 1 \leq i \leq n$, and the canonical volume element of $\mathbf{R}^{n}$ is denoted by $\omega_{0}=d x^{1} \wedge d x^{2} \wedge \ldots \wedge d x^{n}$.

Denote by $T_{n}^{1} Q$ the manifold of $n$-velocities of order 1 over $Q$. Elements of $T_{n}^{1} Q$ are 1-jets $J_{0}^{1} \zeta \in J_{(0, y)}^{1}\left(\mathbf{R}^{n}, Q\right)$ with origin $0 \in \mathbf{R}^{n}$ and target $y=\zeta(0) \in Q$. The canonical projection $\tau_{n}^{1,0}: T_{n}^{1} Q \rightarrow Q$ is defined by $\tau_{n}^{1,0}\left(J_{0}^{1} \zeta\right)=\zeta(0)$. In the standard sense, $T_{n}^{1} Q$ is endowed with the canonical smooth manifold structure: for any chart $(V, \psi), \psi=\left(y^{K}\right), 1 \leq K \leq m+n$, on $Q$, the pair $\left(V_{n}^{1}, \psi_{n}^{1}\right), \psi_{n}^{1}=\left(y^{K}, y_{j}^{K}\right)$, is a chart on $T_{n}^{1} Q$, where $V_{n}^{1}=\left(\tau_{n}^{1,0}\right)^{-1}(V), y_{j}^{K}\left(J_{0}^{1} \zeta\right)=D_{j}\left(y^{K} \zeta\right)(0), 1 \leq j \leq n$, and $\operatorname{dim} T_{n}^{1} Q=(n+m)(n+1)$. Recall that there is a canonical identification of the jet space $J^{1}\left(\mathbf{R}^{n} \times Q\right)$ and the product $\mathbf{R}^{n} \times T_{n}^{1} Q$,

$$
\phi: J^{1}\left(\mathbf{R}^{n} \times Q\right) \rightarrow \mathbf{R}^{n} \times T_{n}^{1} Q
$$

defined by $\phi\left(J_{x}^{1} \gamma\right)=\left(x, J_{0}^{1}\left(\gamma_{0} \circ \operatorname{tr}_{-x}\right)\right)$, where $\gamma_{0}$ is the principal part of $\gamma$, and $\operatorname{tr}_{\alpha}: \mathbf{R}^{n} \rightarrow \mathbf{R}^{n}, \operatorname{tr}_{\alpha}(x)=x-\alpha$, is the translation of the Euclidean space $\mathbf{R}^{n}$.

Suppose $\zeta: U \rightarrow Q$ be a differentiable mapping defined on an open set $U \subset \mathbf{R}^{n}$. The 1-jet prolongation of $\zeta$ is the mapping

$$
U \ni x \rightarrow\left(T_{n}^{1} \zeta\right)(x)=J_{0}^{1}\left(\zeta \circ \operatorname{tr}_{-x}\right) \in T_{n}^{1} Q
$$

Note that for any diffeomorphism $\mu: \bar{U} \rightarrow U$ of open subsets of $\mathbf{R}^{n}$, the prolongation $T_{n}^{1} \zeta$ of $\zeta$ satisfies

$$
T_{n}^{1}(\zeta \circ \mu)(z)=T_{n}^{1}(\zeta)(\mu(z)) \circ \mu^{1}(z)
$$

where $\mu^{1}(z)=J_{0}^{1}\left(\operatorname{tr}_{\mu(z)} \circ \mu \circ \operatorname{tr}_{-z}\right)$. Indeed, by the definition of $T_{n}^{1} \zeta, T_{n}^{1}(\zeta \circ \mu)(z)=$ $J_{0}^{1}\left(\zeta \circ \mu \circ \operatorname{tr}_{-z}\right)=J_{0}^{1}\left(\zeta \circ \operatorname{tr}_{-\mu(z)}\right) \circ J_{0}^{1}\left(\operatorname{tr}_{\mu(z)} \circ \mu \circ \operatorname{tr}_{-z}\right)=T_{n}^{1}(\zeta)(\mu(z)) \circ \mu^{1}(z)$. The identity (3.3) is used to prove the forthcoming Theorem 13

We restrict our attention to mappings which are immersions (i.e. their tangent mappings are injective). $J_{0}^{1} \zeta \in T_{n}^{1} Q$ is called regular, if every representative of $J_{0}^{1} \zeta$ is an immersion at $0 \in \mathbf{R}^{n}$. The set $\operatorname{Imm} T_{n}^{1} Q$ of regular velocities form an open subset of $T_{n}^{1} Q$, and with the open submanifold structure $\operatorname{Imm} T_{n}^{1} Q$ is called the manifold of regular $n$-velocities of order 1 over $Q$. Restricting the coordinates $\psi_{n}^{1}=\left(y^{K}, y_{j}^{K}\right)$, we get the canonical charts on $\operatorname{Imm} T_{n}^{1} Q$, induced by the canonical atlas of $T_{n}^{1} Q$.

The manifold of regular velocities $\operatorname{Imm} T_{n}^{1} Q$ is also endowed with another smooth structures. We denote by $(i)=\left(i_{1}, i_{2}, \ldots, i_{n}\right)$ an increasing $n$-subsequence of the sequence $(1,2, \ldots, m+n)$, and by $(\sigma)$ the complementary increasing subsequence. From the definition of an immersion it follows that for every regular velocity $J_{0}^{1} \zeta \in$ $V_{n}^{1}$ there exists an $n$-subsequence $(i)$ of $(1,2, \ldots, m+n)$ such that $\operatorname{det} y_{j}^{i}\left(J_{0}^{1} \zeta\right)=$ $\operatorname{det}\left(D_{j}\left(y^{i} \zeta\right)(0)\right) \neq 0, i \in(i), 1 \leq j \leq n$. We set

$$
V_{n}^{1(i)}=\left\{J_{0}^{1} \zeta \in V_{n}^{1} \mid \operatorname{det}\left(D_{j}\left(y^{i} \zeta\right)(0)\right) \neq 0\right\},
$$

for every $n$-subsequence $(i)$ of $(1,2, \ldots, m+n)$, where $\left(V_{n}^{1}, \psi_{n}^{1}\right)$ is a chart on $\operatorname{Imm} T_{n}^{1} Q$ associated with $(V, \psi)$. Clearly, $V_{n}^{1(i)}$ is an open subset of $V_{n}^{1}$, and $V_{n}^{1}$ is covered by the sets $V_{n}^{1(i)}$, where $(i)$ runs through all $n$-subsequences of 
$(1,2, \ldots, m+n)$. The charts $\left(V_{n}^{1(i)}, \psi_{n}^{1(i)}\right)$, where $\psi_{n}^{1(i)}$ denotes the canonical coordinates $\psi_{n}^{1}$ on $V_{n}^{1}$ restricted to $V_{n}^{1(i)}$, constitute an atlas on $\operatorname{Imm} T_{n}^{1} Q$ (finer than the canonical atlas). Another smooth atlas on $\operatorname{Imm} T_{n}^{1} Q$, which arise from the canonical charts, is obtained in the following way. To this purpose we introduce a set of functions $z_{i}^{k}: V_{n}^{1(i)} \rightarrow \mathbf{R}$, defined by the formula $z_{i}^{k} y_{j}^{i}=\delta_{j}^{k}$ (the Kronecker symbol), where $i \in(i), 1 \leq j, k \leq n$. For every $\operatorname{chart}\left(V_{n}^{1(i)}, \psi_{n}^{1(i)}\right), \psi_{n}^{1(i)}=\left(y^{i}, y^{\sigma}, y_{j}^{i}, y_{j}^{\sigma}\right)$, on $\operatorname{Imm} T_{n}^{1} Q$ we put

$$
w^{i}=y^{i}, \quad w^{\sigma}=y^{\sigma}, \quad w_{j}^{i}=y_{j}^{i}, \quad w_{i}^{\sigma}=z_{i}^{j} y_{j}^{\sigma},
$$

where $i \in(i), \sigma \in(\sigma), 1 \leq j \leq n$. Formula (3.5) defines charts $\left(V_{n}^{1(i)}, \chi_{n}^{1(i)}\right)$, $\chi_{n}^{1(i)}=\left(w^{i}, w^{\sigma}, w_{j}^{i}, w_{i}^{\sigma}\right)$, which constitute an atlas on $\operatorname{Imm} T_{n}^{1} Q$. Note that the coordinate functions $w_{i}^{\sigma}$ arise as the derivatives $\Delta_{i} w^{\sigma}$ of the base coordinates $w^{\sigma}$, where $\Delta_{i}, i \in(i)$, is the $(i)$-adapted formal derivative morphism over $Q$, expressed by

$$
\Delta_{i}=z_{i}^{j} d_{j}=z_{i}^{j} y_{j}^{K} \frac{\partial}{\partial y^{K}}=z_{i}^{j} y_{j}^{p} \frac{\partial}{\partial y^{p}}+z_{i}^{j} y_{j}^{\sigma} \frac{\partial}{\partial y^{\sigma}}=\frac{\partial}{\partial w^{i}}+w_{i}^{\sigma} \frac{\partial}{\partial w^{\sigma}},
$$

(summation runs through $1 \leq j \leq n, K \in(1,2, \ldots, m+n)$ ).

A particular subset of regular $n$-velocities of order 1 whose both the origin and the target are at the point $0 \in \mathbf{R}^{n}, \operatorname{Imm} J_{(0,0)}^{1}\left(\mathbf{R}^{n}, \mathbf{R}^{n}\right)$, coincides with the general linear group $G L_{n}(\mathbf{R})$, and is endowed with a global chart defined by the coordinate functions $a_{j}^{i}: L_{n}^{1} \rightarrow \mathbf{R}, a_{j}^{i}\left(J_{0}^{1} \alpha\right)=D_{j} \alpha^{i}(0), 1 \leq i, j \leq n$. Note that for an arbitrary finite $r, L_{n}^{r}=\operatorname{Imm} J_{(0,0)}^{r}\left(\mathbf{R}^{n}, \mathbf{R}^{n}\right)$ is the $r$-th differential group of $\mathbf{R}^{n}$ (known from the theory of differential invariants), and $L_{n}^{1}=G L_{n}(\mathbf{R})$. The canonical right action of $G L_{n}(\mathbf{R})$ on $T_{n}^{1} Q$ is defined by means of the jet composition and reduces onto $\operatorname{Imm} T_{n}^{1} Q$,

$$
\operatorname{Imm} T_{n}^{1} Q \times G L_{n}(\mathbf{R}) \ni\left(J_{0}^{1} \zeta, J_{0}^{1} \alpha\right) \rightarrow J_{0}^{1} \zeta \circ J_{0}^{1} \alpha=J_{0}^{1}(\zeta \circ \alpha) \in \operatorname{Imm} T_{n}^{1} Q .
$$

It is easy to observe that the coordinates $w^{i}, w^{\sigma}, w_{i}^{\sigma}$ (3.5) of the chart $\left(V_{n}^{1(i)}, \chi_{n}^{1(i)}\right)$ are $G L_{n}(\mathbf{R})$-invariant. $\left(V_{n}^{1(i)}, \chi_{n}^{1(i)}\right)$ is called the $(i)$-subordinate chart to the chart $(V, \psi)$ on $Q$, adapted to the canonical group action of $G L_{n}(\mathbf{R})$ on $\operatorname{Imm} T_{n}^{1} Q$.

Consider the orbit space with respect to the group action (3.7),

$$
G_{n}^{1} Q=\operatorname{Imm} T_{n}^{1} Q / G L_{n}(\mathbf{R})
$$

with its quotient topology. Elements of $G_{n}^{1} Q$ are classes of regular velocities with respect to the equivalence relation "there exists an element $J_{0}^{1} \alpha \in G L_{n}(\mathbf{R})$ such that $J_{0}^{1} \zeta=J_{0}^{1} \chi \circ J_{0}^{1} \alpha$ " on $\operatorname{Imm} T_{n}^{1} Q$; a class $\left[J_{0}^{1} \zeta\right] \in G_{n}^{1} Q$ is also called a contact element of order 1 on $Q$, represented by an immersion $\zeta$. The quotient projection $\operatorname{Imm} T_{n}^{1} Q \ni J_{0}^{1} \zeta \rightarrow\left[J_{0}^{1} \zeta\right] \in G_{n}^{1} Q$ is denoted by $\kappa_{n}^{1}: \operatorname{Imm} T_{n}^{1} Q \rightarrow G_{n}^{1} Q$, and the canonical projection $\tau_{n, G}^{1,0}: G_{n}^{1} Q \rightarrow Q$ is defined by $\tau_{n, G}^{1,0}\left(\left[J_{0}^{1} \zeta\right]\right)=\zeta(0)$.

The following theorem characterizes the structure of $\operatorname{Imm} T_{n}^{1} Q$ and $G_{n}^{1} Q$.

Theorem 10. Let $Q$ be Hausdorff.

(a) The canonical action of $G L_{n}(\mathbf{R})$ defines on $\operatorname{Imm} T_{n}^{1} Q$ the structure of right principle $G L_{n}(\mathbf{R})$-bundle with base $G_{n}^{1} Q$ and type fibre $\operatorname{Imm} J_{(0,0)}^{r}\left(\mathbf{R}^{n}, \mathbf{R}^{m+n}\right)$.

(b) The orbit space $G_{n}^{1} Q$ has a unique smooth structure such that the canonical quotient projection $\kappa_{n}^{1}$ is a submersion. 
(c) The orbit manifold $G_{n}^{1} Q$ has the structure of a fibration with base $Q$, projection $\tau_{n, G}^{1,0}$, and type fibre $G_{n, n+m}^{1}=\operatorname{Imm} J_{(0,0)}^{r}\left(\mathbf{R}^{n}, \mathbf{R}^{m+n}\right) / G L_{n}(\mathbf{R})$. The dimension of $G_{n}^{1} Q$ equals $\operatorname{dim} G_{n}^{1} Q=m(n+1)+n$.

Proof. See Grigore and Krupka [10.

$G_{n}^{1} Q$ together with the smooth and fibration structure described by Theorem 10 is called the Grassmann fibration of order 1 over the manifold $Q$. A smooth structure on $G_{n}^{1} Q$ is described as follows. Let $\left(V_{n}^{1(i)}, \chi_{n}^{1(i)}\right), \chi_{n}^{1(i)}=\left(w^{i}, w^{\sigma}, w_{j}^{i}, w_{i}^{\sigma}\right)$, be an $(i)$-subordinate chart on $\operatorname{Imm} T_{n}^{1} Q$ to the $\operatorname{chart}(V, \psi)$ on $Q$. Denote $\tilde{V}_{n}^{1(i)}=$ $\kappa_{n}^{1}\left(V_{n}^{1(i)}\right)$, and $\tilde{\chi}_{n}^{1(i)}=\left(\tilde{w}^{i}, \tilde{w}^{\sigma}, \tilde{w}_{i}^{\sigma}\right)$, where

$$
\tilde{w}^{i}\left(\left[J_{0}^{1} \zeta\right]\right)=w^{i}\left(J_{0}^{1} \zeta\right), \quad \tilde{w}^{\sigma}\left(\left[J_{0}^{1} \zeta\right]\right)=w^{\sigma}\left(J_{0}^{1} \zeta\right), \quad \tilde{w}_{i}^{\sigma}\left(\left[J_{0}^{1} \zeta\right]\right)=w_{i}^{\sigma}\left(J_{0}^{1} \zeta\right) .
$$

The chart $\left(\tilde{V}_{n}^{1(i)}, \tilde{\chi}_{n}^{1(i)}\right)$ on $G_{n}^{1} Q$ is the associated chart with $\left(V_{n}^{1(i)}, \chi_{n}^{1(i)}\right)$. Usually we do not distinguish between coordinates on $\operatorname{Imm} T_{n}^{1} Q$ and $G_{n}^{1} Q$, when no misunderstanding may arise.

Let $\zeta: U \rightarrow Q$ be an immersion on an open subset $U$ of $\mathbf{R}^{n}$ with values in $Q$. Recall that the 1 -jet prolongation of $\zeta$, the curve $T_{n}^{1} \zeta: U \rightarrow T_{n}^{1} Q$ is defined by (3.2). An immersion $G_{n}^{1} \zeta: U \rightarrow G_{n}^{1} Q$, given by

$$
\left(G_{n}^{1} \zeta\right)(x)=\left[\left(T_{n}^{1} \zeta\right)(x)\right]
$$

is called the Grassmann prolongation of $\zeta$. If $\mu: \bar{U} \rightarrow U$ is a diffeomorphism of open subsets of $\mathbf{R}^{n}$, then the mapping (3.9) satisfies

$$
G_{n}^{1}(\zeta \circ \mu)=\left(G_{n}^{1} \zeta\right) \circ \mu .
$$

Indeed, using the property (3.3), for any $z \in \bar{U}$ we have

$$
\begin{aligned}
G_{n}^{1}(\zeta \circ \mu)(z) & =\left[T_{n}^{1}(\zeta \circ \mu)(z)\right]=\left[T_{n}^{1}(\zeta)(\mu(z)) \circ \mu^{1}(z)\right] \\
& =\left[T_{n}^{1}(\zeta)(\mu(z))\right]=G_{n}^{1}(\zeta)(\mu(z)) .
\end{aligned}
$$

An arbitrary diffeomorphism $\alpha: W \rightarrow Q$ (onto its image), defined on an open subset $W \subset Q$, can be prolonged on the Grassmann fibration $G_{n}^{1} Q$ as follows. We define a diffeomorphism of $G_{n}^{1} Q, G_{n}^{1} \alpha: W^{1} \rightarrow G_{n}^{1} Q$, where $\tilde{W}^{1}=\left(\tau_{n, G}^{1,0}\right)^{-1}(W)$, by putting

$$
G_{n}^{1} \alpha\left(\left[J_{0}^{1} \zeta\right]\right)=\left[J_{0}^{1}(\alpha \circ \zeta)\right]
$$

for every $J_{0}^{1} \zeta \in \operatorname{Imm} T_{n}^{1} Q$ such that $\alpha$ is composable with $\zeta$. $G_{n}^{1} \alpha$ is called the Grassmann prolongation of $\alpha$. Note that combining these concepts, the prolongation of an immersion and the prolongation of a diffeomorphism, it is easy to see that $G_{n}^{1}(\alpha \circ \zeta)=G_{n}^{1} \alpha \circ G_{n}^{1} \zeta$; for every $x \in U$,

$$
G_{n}^{1}(\alpha \circ \zeta)(x)=\left[J_{0}^{1}\left(\alpha \circ \zeta \circ \operatorname{tr}_{-x}\right)\right]=G_{n}^{1} \alpha\left(\left[J_{0}^{1}\left(\zeta \circ \operatorname{tr}_{-x}\right)\right]\right)=G_{n}^{1} \alpha \circ G_{n}^{1} \zeta(x) .
$$

Consider a vector field $\Xi$ defined on $W \subset Q$, and the local one-parameter group $\alpha_{t}^{\Xi}$ of $\Xi . G_{n}^{1} \alpha_{t}^{\Xi}$ is the Grassmann prolongation of $\alpha_{t}^{\Xi}(3.10)$. We define

$$
G_{n}^{1} \Xi\left(\left[J_{0}^{1} \zeta\right]\right)=\left(\frac{d}{d t} G_{n}^{1} \alpha_{t}^{\Xi}\left(\left[J_{0}^{1} \zeta\right]\right)\right)_{0}
$$

for every element $\left[J_{0}^{1} \zeta\right] \in \tilde{W}^{1} \subset G_{n}^{1} Q$. Formula (3.12) defines a vector field $G^{1} \Xi$ on an open subset $W^{1}$, called the Grassmann prolongation of vector field $\Xi$. 
Lemma 11. If a vector field $\Xi$ on $W \subset Q$ has an expression

$$
\Xi=\Xi^{K} \frac{\partial}{\partial y^{K}}
$$

with respect to a chart $(V, \psi), \psi=\left(y^{K}\right)$, such that $V \subset W$, then the Grassmann prolongation $G_{n}^{1} \Xi$ is expressed with respect to the $(i)$-subordinate $\operatorname{chart}\left(\tilde{V}_{n}^{1(i)}, \tilde{\chi}_{n}^{1(i)}\right)$ (3.8) as

$$
G_{n}^{1} \Xi=\Xi^{i} \frac{\partial}{\partial w^{i}}+\Xi^{\sigma} \frac{\partial}{\partial w^{\sigma}}+\Xi_{i}^{\sigma} \frac{\partial}{\partial w_{i}^{\sigma}}
$$

where $\Xi^{K}=\Xi^{K}\left(w^{i}, w^{\sigma}\right), \Xi_{i}^{\sigma}=\Xi_{i}^{\sigma}\left(w^{i}, w^{\sigma}, w_{i}^{\sigma}\right)$, and

$$
\Xi_{i}^{\sigma}=\Delta_{i} \Xi^{\sigma}-w_{p}^{\sigma} \Delta_{i} \Xi^{p} .
$$

Proof. By the definition of $G_{n}^{1} \Xi$ (3.12),

$$
\Xi_{i}^{\sigma}\left(\left[J_{0}^{1} \zeta\right]\right)=\left(\frac{d}{d t} w_{i}^{\sigma} \circ G_{n}^{1} \alpha_{t}^{\Xi}\left(\left[J_{0}^{1} \zeta\right]\right)\right)_{0},
$$

where we differentiate the function $t \rightarrow w_{i}^{\sigma} \circ G_{n}^{1} \alpha_{t}^{\Xi}\left(\left[J_{0}^{1} \zeta\right]\right)=\Delta_{i} w^{\sigma}\left(J_{0}^{1}\left(\alpha_{t}^{\Xi} \circ \zeta\right)\right)$ at $t=0$, where $\Delta_{i}=z_{i}^{j} d_{j}$ is the (i)-adapted formal derivative morphism (3.6). Using the chart transformation (3.5), a straightforward calculation at a point $J_{0}^{1} \zeta$ now gives

$$
\begin{aligned}
& \Xi_{i}^{\sigma}\left(\left[J_{0}^{1} \zeta\right]\right)=\left(\frac{d}{d t} z_{i}^{j}\left(J_{0}^{1}\left(\alpha_{t}^{\Xi} \circ \zeta\right)\right)\right)_{0} d_{j} w^{\sigma}+z_{i}^{j}\left(\frac{d}{d t} d_{j} w^{\sigma}\left(J_{0}^{1}\left(\alpha_{t}^{\Xi} \circ \zeta\right)\right)\right)_{0} \\
& \left.\left.=\frac{\partial z_{i}^{j}}{\partial y_{k}^{p}}\left(\frac{d}{d t} D_{k}\left(y^{p} \alpha_{t}^{\Xi} \psi^{-1} \circ \psi \zeta\right)\right)\right)_{0} y_{j}^{\sigma}+z_{i}^{j}\left(\frac{d}{d t} D_{j}\left(y^{\sigma} \alpha_{t}^{\Xi} \psi^{-1} \circ \psi \zeta\right)(0)\right)\right)_{0} \\
& =-z_{p}^{j} z_{i}^{k} y_{j}^{\sigma} y_{k}^{L}\left(\frac{d}{d t} D_{L}\left(y^{p} \alpha_{t}^{\Xi} \psi^{-1}\right)(\psi \zeta(0))\right)_{0}+z_{i}^{j} y_{j}^{L}\left(\frac{d}{d t} D_{L}\left(y^{\sigma} \alpha_{t}^{\Xi} \psi^{-1}\right)(\psi \zeta(0))\right)_{0} \\
& =-\delta_{i}^{s} w_{p}^{\sigma}\left(\frac{\partial \Xi^{p}}{\partial y^{s}}\right)_{\zeta(0)}-w_{i}^{\nu} w_{p}^{\sigma}\left(\frac{\partial \Xi^{p}}{\partial y^{\nu}}\right)_{\zeta(0)}+\delta_{i}^{s}\left(\frac{\partial \Xi^{\sigma}}{\partial y^{s}}\right)_{\zeta(0)}+w_{i}^{\nu}\left(\frac{\partial \Xi^{\sigma}}{\partial y^{\nu}}\right)_{\zeta(0)} \\
& =\left(\frac{\partial \Xi^{\sigma}}{\partial y^{i}}\right)_{\zeta(0)}+w_{i}^{\nu}\left(\frac{\partial \Xi^{\sigma}}{\partial y^{\nu}}\right)_{\zeta(0)}-w_{p}^{\sigma}\left(\frac{\partial \Xi^{p}}{\partial y^{i}}\right)_{\zeta(0)}-w_{p}^{\sigma} w_{i}^{\nu}\left(\frac{\partial \Xi^{p}}{\partial y^{\nu}}\right)_{\zeta(0)} \\
& =\Delta_{i} \Xi^{\sigma}-w_{p}^{\sigma} \Delta_{i} \Xi^{p},
\end{aligned}
$$

proving (3.13).

Remark 12. Lemma 11 is a modification of the standard concept of a jet prolongation of a projectable vector field, defined on a fibered manifold. If $\Xi$ is a vector field on $T_{n}^{1} Q$ (embedded in $\mathbf{R}^{n} \times T_{n}^{1} Q$ ), expressed by $\Xi=\Xi^{K}\left(\partial / \partial y^{K}\right)$, then the jet prolongation $J^{1} \Xi$, resp. $J^{2} \Xi$, of $\Xi$ has the expression

$$
J^{1} \Xi=\Xi^{K} \frac{\partial}{\partial y^{K}}+\Xi_{j}^{K} \frac{\partial}{\partial y_{j}^{K}}, \text { resp. } J^{2} \Xi=\Xi^{K} \frac{\partial}{\partial y^{K}}+\Xi_{j}^{K} \frac{\partial}{\partial y_{j}^{K}}+\Xi_{j l}^{K} \frac{\partial}{\partial y_{j l}^{K}},
$$

where $\Xi_{j}^{K}=d_{j} \Xi^{K}, \Xi_{j l}^{K}=d_{l} \Xi_{j}^{K}$.

A differential form $\eta$ on $\tilde{W}^{1} \subset G_{n}^{1} Q$ is called contact, if $\left(G_{n}^{1} \zeta\right)^{*} \eta=0$ for all immersions $\zeta: U \rightarrow W$, defined on an open subset $U$ of $\mathbf{R}^{n}$. If $(V, \psi), \psi=\left(y^{K}\right)$, 
is a chart on $Q$ such that $V \subset W$, and $\left(\tilde{V}_{n}^{1(i)}, \tilde{\chi}_{n}^{1(i)}\right), \tilde{\chi}_{n}^{1(i)}=\left(w^{i}, w^{\sigma}, w_{i}^{\sigma}\right)$, is the (i)-subordinate chart on $G_{n}^{1} Q$, then the 1 -forms $d w^{i}, \tilde{\omega}^{\sigma}, d w_{i}^{\sigma}$, where

$$
\tilde{\omega}^{\sigma}=d w^{\sigma}-w_{i}^{\sigma} d w^{i}
$$

(sum through $i \in(i)$ ), constitute a basis of linear forms on $\tilde{V}_{n}^{1(i)}$. Differential forms, which are locally generated by contact 1 -forms $\tilde{\omega}^{\sigma}$ and 2 -forms $d \tilde{\omega}^{\sigma}$, constitute the contact ideal $\tilde{\Theta}^{1} W$ in the exterior algebra of differential forms on $\tilde{W}^{1}$. Using the definition of charts on $G_{n}^{1} Q$, and the canonical embedding of $\operatorname{Imm} T_{n}^{1} Q$ into $J^{1}\left(\mathbf{R}^{n} \times Q\right)$, we note that $\tilde{\omega}^{\sigma}$ can be expressed as a linear combination of contact 1-forms $\omega^{K}=d y^{K}-y_{l}^{K} d x^{l}$ (2.1) on $J^{1}\left(\mathbf{R}^{n} \times Q\right)$,

$$
\begin{aligned}
\left(\pi^{2,1}\right)^{*} \tilde{\omega}^{\sigma} & =\left(\pi^{2,1}\right)^{*}\left(d y^{\sigma}-z_{i}^{j} y_{j}^{\sigma} d y^{i}\right) \\
& =\omega^{\sigma}+y_{l}^{\sigma} d x^{l}-z_{i}^{j} y_{j}^{\sigma}\left(\omega^{i}+y_{l}^{i} d x^{l}\right)=\omega^{\sigma}-z_{i}^{j} y_{j}^{\sigma} \omega^{i} .
\end{aligned}
$$

\section{The fundamental Lepage equivalent of a homogeneous Lagrangian}

First we recall the notion of a positive homogeneous function and study its properties. This is defined as an equivariance with respect to the canonical right action of the identity component $G L_{n}^{+}(\mathbf{R})$ of the general linear group $G L_{n}(\mathbf{R})$. We say that a real-valued function $F: \operatorname{Imm} T_{n}^{1} Q \rightarrow \mathbf{R}$ is positive homogeneous, if

$$
F\left(J_{0}^{1} \zeta \circ J_{0}^{1} \alpha\right)=\operatorname{det}\left(a_{j}^{i}\left(J_{0}^{1} \alpha\right)\right) F\left(J_{0}^{1} \zeta\right)
$$

for all $J_{0}^{1} \zeta \in \operatorname{Imm} T_{n}^{1} Q$ and all $J_{0}^{1} \alpha \in G L_{n}^{+}(\mathbf{R})$. Note that the elements of $G L_{n}(\mathbf{R})$ used in this definition are represented by orientation-preserving diffeomorphisms.

Let $U$ be an open subset of $\mathbf{R}^{n}$, and let $\zeta: U \rightarrow Q$ be an immersion. Any compact subset $S$ of $U$ associates with a function $F: \operatorname{Imm} T_{n}^{1} Q \rightarrow \mathbf{R}$ the integral

$$
F_{S}(\zeta)=\int_{S}\left(F \circ T_{n}^{1} \zeta\right) \omega_{0}
$$

The following theorem is a criterion of parameter-invariance of this integral.

Theorem 13. (Euler-Zermelo) Let $F: \operatorname{Imm} T_{n}^{1} Q \rightarrow \mathbf{R}$ be a differentiable function. The following conditions are equivalent:

(a) $F$ is positive homogeneous.

(b) The integral (4.1) is parameter-invariant, i.e. if $\zeta: U \rightarrow Q$ is an immersion, and $\mu: \bar{U} \rightarrow U$ is a diffeomorphism of open subsets in $\mathbf{R}^{n}$ such that $\operatorname{det} D \mu>0$ on $\bar{U}$, then for any two compact subsets $\bar{U}_{0} \subset \bar{U}, U_{0} \subset U$, such that $\mu\left(\bar{U}_{0}\right)=U_{0}$, the integral (4.1) satisfies $F_{U_{0}}(\zeta)=F_{\bar{U}_{0}}(\zeta \circ \mu)$.

(c) For any chart $(V, \psi), \psi=\left(y^{K}\right)$, on $Q$,

$$
\frac{\partial F}{\partial y_{j}^{K}} y_{l}^{K}=\delta_{l}^{j} F, \quad j, l=1,2, \ldots, n .
$$

Proof. The proof for $n=1$ can be found in Urban and Krupka [24]; for arbitrary positive integer $n$ the proof proceeds along the same lines.

Remark 14. The identities (4.2) are the well-known Zermelo conditions (cf. McKiernan [19]). Differentiating (4.2) with respect to $y_{j}^{K}$, we obtain the following 
formulas for partial derivatives of a positive homogeneous function $F$. Namely, for an integer $k \geq 1$ we have

$$
\begin{array}{r}
\frac{\partial^{k} F}{\partial y_{j_{1}}^{K_{1}} \partial y_{j_{2}}^{K_{2}} \ldots \partial y_{j_{k}}^{K_{k}}} y_{i_{1}}^{K_{1}}=\frac{\partial^{k-1} F}{\partial y_{j_{2}}^{K_{2}} \partial y_{j_{3}}^{K_{3}} \ldots \partial y_{j_{k}}^{K_{k}}} \delta_{i_{1}}^{j_{1}}-\frac{\partial^{k-1} F}{\partial y_{j_{1}}^{K_{2}} \partial y_{j_{3}}^{K_{3}} \ldots \partial y_{j_{k}}^{K_{k}}} \delta_{i_{1}}^{j_{2}} \\
-\frac{\partial^{k-1} F}{\partial y_{j_{2}}^{K_{2}} \partial y_{j_{1}}^{K_{3}} \partial y_{j_{4}}^{K_{4}} \ldots \partial y_{j_{k}}^{K_{k}}} \delta_{i_{1}}^{j_{3}}-\ldots-\frac{\partial^{k-1} F}{\partial y_{j_{2}}^{K_{2}} \partial y_{j_{3}}^{K_{3}} \ldots \partial y_{j_{1}}^{K_{k}}} \delta_{i_{1}}^{j_{k}}
\end{array}
$$

Remark 15. On the chart neighborhood $V_{n}^{1(i)}$ (3.4), the Zermelo conditions (4.2) for the function $\tilde{F}=F \circ\left(\psi_{n}^{1(i)}\right)^{-1} \circ \chi_{n}^{1(i)}$ reads

$$
\frac{\partial \tilde{F}}{\partial w_{j}^{i}} w_{k}^{i}=\delta_{k}^{j} \tilde{F}
$$

where summation runs through $i \in(i)$. It is easy to see that this equation can be integrated and its solution is of the form

$$
\tilde{F}\left(w^{i}, w^{\sigma}, w_{j}^{i}, w_{i}^{\sigma}\right)=\operatorname{det}\left(w_{j}^{i}\right) \tilde{F}_{G}\left(w^{i}, w^{\sigma}, w_{i}^{\sigma}\right),
$$

where $\tilde{F}_{G}$ is a uniquely given differentiable function on $\tilde{V}_{n}^{1(i)} \subset G_{n}^{1} Q$. We call $\tilde{F}_{G}$ the Grassmann projection of the (positive homogeneous) function $F$, associated to $\left(\tilde{V}_{n}^{1(i)}, \tilde{\chi}_{n}^{1(i)}\right)$.

Lemma 16. Let $F: \operatorname{Imm} T_{n}^{1} Q \rightarrow \mathbf{R}$ be a positive homogeneous function. Then for every integer $k, 0 \leq k \leq n-1$, and every integer $l$ such that, $k<l \leq n$,

$$
\frac{\partial^{l} F}{\partial y_{j_{1}}^{K_{1}} \ldots \partial y_{j_{l}}^{K_{l}}} y_{i_{k+1}}^{K_{k+1}} \ldots y_{i_{l}}^{K_{l}} \varepsilon_{j_{1} \ldots j_{l} i_{l+1} \ldots i_{n}}=\frac{l !}{k !} \frac{\partial^{k} F}{\partial y_{j_{1}}^{K_{1}} \ldots \partial y_{j_{k}}^{K_{k}}} \varepsilon_{j_{1} \ldots j_{k} i_{k+1} \ldots i_{n}} .
$$

Proof. Formula (4.5) follows from a direct application of (4.3) in $(l-k)$ steps, and the skew-symmetry property of the Levi-Civita symbol. Indeed, we have

$$
\begin{aligned}
& \frac{\partial^{l} F}{\partial y_{j_{1}}^{K_{1}} \ldots y_{j_{k}}^{K_{k}} y_{j_{k+1}}^{K_{k+1}} \ldots \partial y_{j_{l}}^{K_{l}}} y_{i_{k+1}}^{K_{k+1}} \ldots y_{i_{l}}^{K_{l}} \varepsilon_{j_{1} \ldots j_{l} i_{l+1} \ldots i_{n}} \\
& =l \frac{\partial^{l-1} F}{\partial y_{j_{1}}^{K_{1}} \ldots y_{j_{k}}^{K_{k}} y_{j_{k+1}}^{K_{k+1}} \ldots \partial y_{j_{l-1}}^{K_{l-1}}} y_{i_{k+1}}^{K_{k+1}} \ldots y_{i_{l-1}}^{K_{l-1}} \varepsilon_{j_{1} \ldots j_{l-1} i_{l} \ldots i_{n}} \\
& =l(l-1) \frac{\partial^{l-2} F}{\partial y_{j_{1}}^{K_{1}} \ldots y_{j_{k}}^{K_{k}} y_{j_{k+1}}^{K_{k+1}} \ldots \partial y_{j_{l-2}}^{K_{l-2}}} y_{i_{k+1}}^{K_{k+1}} \ldots y_{i_{l-2}}^{K_{l-2}} \varepsilon_{j_{1} \ldots j_{l-2} i_{l-1} i_{l} \ldots i_{n}} \\
& =\ldots=l(l-1)(l-2) \ldots(k+2)(k+1) \frac{\partial^{k} F}{\partial y_{j_{1}}^{K_{1}} \ldots \partial y_{j_{k}}^{K_{k}}} \varepsilon_{j_{1} \ldots j_{k} i_{k+1} \ldots i_{n}},
\end{aligned}
$$

as required.

In the following theorem we study the fundamental Lepage equivalent $Z_{\lambda}$ (2.4) of a first-order Lagrangian $\lambda \in \Omega_{n, \mathbf{R}^{n}}^{1}\left(\mathbf{R}^{n} \times Q\right), \lambda=\mathscr{L} \omega_{0}$, whose Lagrange function $\mathscr{L}$, defined on the manifold of regular velocities $\operatorname{Imm} T_{n}^{1} Q$, is positive-homogeneous. We derive its local structure and show, in particular, that the resulting $n$-form is defined on the Grassmann fibration $G_{n}^{1} Q$. 
Theorem 17. Let $\lambda \in \Omega_{n, \mathbf{R}^{n}}^{1}\left(\mathbf{R}^{n} \times Q\right)$ be a Lagrangian of order 1 , expressed by $\lambda=\mathscr{L} \omega_{0}$, where $\mathscr{L}: \operatorname{Imm} T_{n}^{1} Q \rightarrow \mathbf{R}$ is the Lagrange function. If $\mathscr{L}$ satisfies the Zermelo condition (4.2), then the fundamental Lepage equivalent (2.4) is defined on the $n$-Grassmann fibration $G_{n}^{1} Q$, and has the expression

$$
W_{\lambda}=\frac{1}{(n !)^{2}} \frac{\partial^{n} \mathscr{L}}{\partial y_{j_{1}}^{K_{1}} \partial y_{j_{2}}^{K_{2}} \ldots \partial y_{j_{n}}^{K_{n}}} \varepsilon_{j_{1} j_{2} \ldots j_{n}} d y^{K_{1}} \wedge d y^{K_{2}} \wedge \ldots \wedge d y^{K_{n}}
$$

Proof. Suppose $\mathscr{L}$ satisfies the Zermelo condition (4.2) hence also identities (4.5). By Lemma 9, the fundamental Lepage equivalent (2.4) can be expressed as

$$
Z_{\lambda}=\sum_{k=0}^{n} \frac{1}{(n-k) !} \frac{1}{k !} Z_{K_{1} \ldots K_{k} i_{k+1} \ldots i_{n}} d y^{K_{1}} \wedge \ldots \wedge d y^{K_{k}} \wedge d x^{i_{k+1}} \wedge \ldots \wedge d x^{i_{n}}
$$

where

$$
\begin{gathered}
Z_{K_{1} \ldots K_{k} i_{k+1} \ldots i_{n}}=\sum_{l=k}^{n}(-1)^{l-k}\left(\begin{array}{c}
n-k \\
n-l
\end{array}\right) \frac{1}{l !} \frac{\partial^{l} \mathscr{L}}{\partial y_{j_{1}}^{K_{1}} \ldots \partial y_{j_{l}}^{K_{l}}} \varepsilon_{j_{1} \ldots j_{l} i_{l+1} \ldots i_{n}} \\
\cdot y_{i_{k+1}}^{K_{k+1}} \ldots y_{i_{l}}^{K_{l}} \operatorname{Alt}\left(i_{k+1}, \ldots, i_{n}\right) .
\end{gathered}
$$

We claim that $Z_{\lambda}$ coincides with $W_{\lambda}$ (4.6). Clearly, the term of $Z_{\lambda}$ containing the exterior product $d y^{K_{1}} \wedge \ldots \wedge d y^{K_{n}}$ reads

$$
\frac{1}{n !} Z_{K_{1} \ldots K_{n}} d y^{K_{1}} \wedge \ldots \wedge d y^{K_{n}}=\frac{1}{n !}\left(\frac{1}{n !} \frac{\partial^{n} \mathscr{L}}{\partial y_{j_{1}}^{K_{1}} \ldots \partial y_{j_{n}}^{K_{n}}} \varepsilon_{j_{1} \ldots j_{n}}\right) d y^{K_{1}} \wedge \ldots \wedge d y^{K_{n}}
$$

which coincides with (4.6). Let us show that all terms of $Z_{\lambda}$ (4.7), which contain exactly $k$ exterior factors $d y^{K}$ vanish whenever $0 \leq k \leq n-1$. Indeed, consider the term of $Z_{\lambda}$ containing the exterior product $d y^{K_{1}} \wedge \ldots \wedge d y^{K_{k}}$ for fixed $k, 0 \leq k \leq$ $n-1$, whose coefficients are given by (4.8). Applying Lemma 16, we obtain

$$
\begin{aligned}
Z_{K_{1} \ldots K_{k} i_{k+1} \ldots i_{n}} & =\sum_{l=k}^{n}(-1)^{l-k}\left(\begin{array}{c}
n-k \\
n-l
\end{array}\right) \frac{1}{l !} \frac{l !}{k !} \frac{\partial^{k} \mathscr{L}}{\partial y_{j_{1}}^{K_{1}} \partial y_{j_{2}}^{K_{2}} \ldots \partial y_{j_{k}}^{K_{k}}} \varepsilon_{j_{1} \ldots j_{k} i_{k+1} \ldots i_{n}} \\
& =\frac{1}{k !} \frac{\partial^{k} \mathscr{L}}{\partial y_{j_{1}}^{K_{1}} \partial y_{j_{2}}^{K_{2}} \ldots \partial y_{j_{k}}^{K_{k}}} \varepsilon_{j_{1} \ldots j_{k} i_{k+1} \ldots i_{n}} \sum_{l=k}^{n}(-1)^{l-k}\left(\begin{array}{c}
n-k \\
n-l
\end{array}\right)=0,
\end{aligned}
$$

as required.

It remains to show that $W_{\lambda}$ (4.6) is defined on $G_{n}^{1} Q$. This is, however, a consequence of positive homogeneity of the Lagrange function $\mathscr{L}$ and properties of the coordinate transformation $\psi_{n}^{1(i)} \circ\left(\chi_{n}^{1(i)}\right)^{-1}$. Indeed, expressing the $n$-form (4.6) in terms of the $G L_{n}(\mathbf{R})$-adapted $(i)$-subordinate chart $\left(V_{n}^{1(i)}, \chi_{n}^{1(i)}\right)$ on $\operatorname{Imm} T_{n}^{1} Q$, arbitrary term of $W_{\lambda}$, up to a coefficient, can be written as

$$
\begin{gathered}
\frac{\partial^{n-l} \tilde{\mathscr{L}}}{\partial w_{i_{l+1}}^{\sigma_{l+1}} \ldots \partial w_{i_{k}}^{\sigma_{k}} \partial w_{i_{k+1}}^{\sigma_{k+1}} \ldots \partial w_{i_{n}}^{\sigma_{n}}}\left|\begin{array}{cccc}
z_{i_{1}}^{j_{1}} & z_{i_{2}}^{j_{1}} & & z_{i_{n}}^{j_{1}} \\
z_{i_{1}}^{j_{2}} & z_{i_{2}}^{j_{2}} & & z_{i_{n}}^{j_{2}} \\
& & \ddots & \\
z_{i_{1}}^{j_{n}} & z_{i_{2}}^{j_{n}} & & z_{i_{n}}^{j_{n}}
\end{array}\right| \\
w_{p_{l+1}}^{\sigma_{l+1}} \ldots w_{p_{k}}^{\sigma_{k}} \delta_{p_{1}}^{i_{1}} \ldots \delta_{p_{l}}^{i_{l}} \varepsilon_{j_{1} j_{2} \ldots j_{n}} d w^{p_{1}} \wedge \ldots \wedge d w^{p_{k}} \wedge \tilde{\omega}^{\sigma_{k+1}} \wedge \ldots \wedge \tilde{\omega}^{\sigma_{n}},
\end{gathered}
$$


for some integers $k, l$ such that $0 \leq k \leq n, 0 \leq l \leq k$, where $\tilde{\mathscr{L}}=\mathscr{L} \circ\left(\psi_{n}^{1(i)}\right)^{-1} \circ$ $\chi_{n}^{1(i)}$, and $\tilde{\omega}^{\sigma}=d w^{\sigma}-w_{i}^{\sigma} d w^{i}($ (3.14) $)$ are contact 1-forms on $\tilde{V}_{n}^{1(i)}$ (cf. Urban and Krupka [21]). Since the determinant $\operatorname{det}\left(z_{i}^{j}\right)$, where $i \in(i), 1 \leq j \leq n$, does not depend on the coordinates $w_{i}^{\sigma}$, we obtain by Remark 15, (4.4), that the function $\tilde{\mathscr{L}} \operatorname{det}\left(z_{i}^{j}\right)$ coincides with the Grassmann projection $\tilde{\mathscr{L}}_{G}$ of $\mathscr{L}$. Hence $W_{\lambda}$ is defined on the neighborhood $\tilde{V}_{n}^{1(i)} \subset G_{n}^{1} Q$ for every chart $(V, \psi)$ on $Q$. This completes the proof.

Remark 18. (Hilbert-Carathéodory form) Analogously, Crampin and Saunders 4 studied the Carathéodory form (2.5) for a positive-homogeneous non-vanishing Lagrange function, resulting into the Hilbert-Carathéodory form, expressed by

$$
\Lambda_{\lambda}=\frac{1}{n !} \frac{1}{\mathscr{L}^{n-1}} \frac{\partial \mathscr{L}}{\partial y_{j_{1}}^{K_{1}}} \frac{\partial \mathscr{L}}{\partial y_{j_{2}}^{K_{2}}} \ldots \frac{\partial \mathscr{L}}{\partial y_{j_{n}}^{K_{n}}} \varepsilon_{j_{1} j_{2} \ldots j_{n}} d y^{K_{1}} \wedge d y^{K_{2}} \wedge \ldots \wedge d y^{K_{n}}
$$

We point out that $\Lambda_{\lambda}$ (4.9) is different from the $n$-form $W_{\lambda}$ (4.6): a simple example for $n=2$ is the Lagrangian $\lambda=\mathscr{L} \omega_{0}$ with positive-homogeneous function $\mathscr{L}=$ $A_{P Q} y_{1}^{P} y_{2}^{Q}$, where functions $A_{P Q}=A_{P Q}\left(y^{K}\right)$ are skew-symmetric; see also Crampin and Saunders [5]. On the other hand, these two Lepage equivalents are closely related as they coincide for the Lagrangian of minimal submanifolds problem (see Section 6).

\section{First-ORDER VARIATIONAL FIELD THEORY FOR SUBMANIFOLDS}

5.1. Lepage forms on $\operatorname{Imm} T_{n}^{1} Q$ and the Euler-Lagrange form. Based on the canonical identification of $J^{1}\left(\mathbf{R}^{n} \times Q\right)$ and $\mathbf{R}^{n} \times T_{n}^{1} Q$ (see (3.1)), we say that an $n$-form $\rho$ on $\operatorname{Imm} T_{n}^{1} Q$ is a Lepage form, if $\rho$ is a Lepage form on $J^{1}\left(\mathbf{R}^{n} \times Q\right)$, i.e. if $h i_{\xi} d \rho=0$ for an arbitrary $\pi^{1,0}$-vertical vector field $\xi$ on $\left(\pi^{1,0}\right)^{-1}(W)$, where $W \subset \mathbf{R}^{n} \times Q$ is an open set, and $\pi^{1,0}: J^{1}\left(\mathbf{R}^{n} \times Q\right) \rightarrow \mathbf{R}^{n} \times Q$ is the canonical jet projection.

Restrict ourselves to $\pi^{1,0}$-horizontal $n$-forms on $\operatorname{Imm} T_{n}^{1} Q$, expressed in any chart $(V, \psi)$ on $Q$ by

$$
\rho=\frac{1}{n !} A_{K_{1} K_{2} \ldots K_{n}} d y^{K_{1}} \wedge d y^{K_{2}} \wedge \ldots \wedge d y^{K_{n}},
$$

where the coefficients $A_{K_{1} K_{2} \ldots K_{n}}$ are functions on $\operatorname{Imm} T_{n}^{1} Q$. Using the canonical decomposition formula $\rho=h \rho+\sum_{k=1}^{n} p_{k} \rho$, we get

$$
h \rho=\mathscr{L} \omega_{0}
$$

where

$$
\mathscr{L}=\frac{1}{n !} A_{K_{1} \ldots K_{n}} y_{j_{1}}^{K_{1}} \ldots y_{j_{n}}^{K_{n}} \varepsilon^{j_{1} \ldots j_{n}}
$$

is a function on $\operatorname{Imm} T_{n}^{1} Q$, and

$$
p_{k} \rho=\frac{1}{k !(n-k) !} B_{K_{1} \ldots K_{k} j_{k+1} \ldots j_{n}} \omega^{K_{1}} \wedge \ldots \wedge \omega^{K_{k}} \wedge d x^{j_{k+1}} \wedge \ldots \wedge d x^{j_{n}},
$$

where

$$
B_{K_{1} \ldots K_{k} j_{k+1} \ldots j_{n}}=A_{K_{1} \ldots K_{n}} y_{j_{k+1}}^{K_{k+1}} \ldots y_{j_{n}}^{K_{n}} \quad \operatorname{Alt}\left(j_{k+1}, \ldots, j_{n}\right) .
$$

The $n$-form $h \rho$ (5.2) is said to be the Lagrangian, and its component $\mathscr{L}(5.3)$ the Lagrange function, associated to $\rho$. If $\rho$ is a Lepage form, $\rho$ is also said to be a Lepage equivalent of the Lagrangian $\lambda=h \rho$. 
Now we give a characterization of Lepage forms on $\operatorname{Imm} T_{n}^{1} Q$.

Theorem 19. Let $(V, \psi)$ be a chart on $Q$. Suppose $\rho$ is a $\pi^{1,0}$-horizontal $n$-form on $\operatorname{Imm} T_{n}^{1} Q$, expressed by (5.1). Then $\rho$ is a Lepage form if and only if

$$
\frac{\partial A_{K_{1} K_{2} \ldots K_{n}}}{\partial y_{s}^{P}} y_{j_{1}}^{K_{1}} y_{j_{2}}^{K_{2}} \ldots y_{j_{n}}^{K_{n}} \varepsilon^{j_{1} j_{2} \ldots j_{n}}=0 .
$$

Proof. We apply the criterion for Lepage forms on $J^{1}\left(\mathbf{R}^{n} \times Q\right)$. If $\rho$ is expressed by (5.1), we get from (5.4), in particular,

$$
\begin{aligned}
p_{1} \rho & =\frac{1}{1 !(n-1) !} A_{K_{1} \ldots K_{n}} y_{j_{2}}^{K_{2}} \ldots y_{j_{n}}^{K_{n}} \omega^{K_{1}} \wedge d x^{j_{2}} \wedge \ldots \wedge d x^{j_{n}} \\
& =\frac{1}{1 !(n-1) !} A_{K K_{2} \ldots K_{n}} y_{j_{2}}^{K_{2}} \ldots y_{j_{n}}^{K_{n}} \varepsilon^{j j_{2} \ldots j_{n}} \omega^{K} \wedge \omega_{j},
\end{aligned}
$$

where $\omega^{K}=d y^{K}-y_{j}^{K} d x^{j}$ and $\omega_{j}=i_{\partial / \partial x^{j}} \omega_{0}$. By Theorem 1 the form $\rho$ is a Lepage form, or the Lepage equivalent of $h \rho=\mathscr{L} \omega_{0}$, where $\mathscr{L}$ is given by (5.3), if and only if the principal component $\Theta$ of $\rho$ has the expression

$$
\Theta=\mathscr{L} \omega_{0}+\frac{\partial \mathscr{L}}{\partial y_{j}^{K}} \omega^{K} \wedge \omega_{j}
$$

This means, however, that $\rho$ is a Lepage form if and only if

$$
\frac{1}{1 !(n-1) !} A_{K K_{2} \ldots K_{n}} y_{j_{2}}^{K_{2}} \ldots y_{j_{n}}^{K_{n}} \varepsilon^{j j_{2} \ldots j_{n}}=\frac{\partial \mathscr{L}}{\partial y_{j}^{K}}
$$

Differentiating $\mathscr{L}$ (5.3), we have

$$
\begin{aligned}
& \frac{\partial \mathscr{L}}{\partial y_{j}^{K}}=\frac{1}{n !} \frac{\partial A_{K_{1} \ldots K_{n}}}{\partial y_{j}^{K}} y_{j_{1}}^{K_{1}} \ldots y_{j_{n}}^{K_{n}} \varepsilon^{j_{1} \ldots j_{n}}+\frac{1}{n !} A_{K_{1} \ldots K_{n}} \frac{\partial}{\partial y_{j}^{K}}\left(y_{j_{1}}^{K_{1}} \ldots y_{j_{n}}^{K_{n}}\right) \varepsilon^{j_{1} \ldots j_{n}} \\
& \quad=\frac{1}{n !} \frac{\partial A_{K_{1} \ldots K_{n}}}{\partial y_{j}^{K}} y_{j_{1}}^{K_{1}} \ldots y_{j_{n}}^{K_{n}} \varepsilon^{j_{1} \ldots j_{n}} \\
& +\frac{1}{n !} A_{K K_{2} \ldots K_{n}} y_{j_{2}}^{K_{2}} \ldots y_{j_{n}}^{K_{n}} \varepsilon^{j j_{2} \ldots j_{n}}+\frac{1}{n !} A_{K_{1} K K_{3} \ldots K_{n}} y_{j_{1}}^{K_{1}} y_{j_{3}}^{K_{3}} \ldots y_{j_{n}}^{K_{n}} \varepsilon^{j_{1} j j_{3} \ldots j_{n}} \\
& \quad+\ldots+\frac{1}{n !} A_{K_{1} \ldots K_{n-1} K} y_{j_{1}}^{K_{1}} \ldots y_{j_{n-1}}^{K_{n-1}} \varepsilon^{j_{1} \ldots j_{n-1} j} \\
& =\frac{1}{n !} \frac{\partial A_{K_{1} \ldots K_{n}}}{\partial y_{j}^{K}} y_{j_{1}}^{K_{1}} \ldots y_{j_{n}}^{K_{n}} \varepsilon^{j_{1} \ldots j_{n}}+\frac{1}{(n-1) !} A_{K K_{2} \ldots K_{n}} y_{j_{2}}^{K_{2}} \ldots y_{j_{n}}^{K_{n}} \varepsilon^{j j_{2} \ldots j_{n}} .
\end{aligned}
$$

Substituting now this expression on the right-hand side of (5.6), we get the formula (5.5). This completes the proof.

Corollary 20. If $\rho$ is a Lepage form on $\operatorname{Imm} T_{n}^{1} Q$, then $h \rho=\mathscr{L} \omega_{0}$, where the function $\mathscr{L}: \operatorname{Imm} T_{n}^{1} Q \rightarrow \mathbf{R}$ is positive homogeneous.

Proof. Suppose $\rho$ is expressed by (5.1) with respect to a chart $(V, \psi)$ on $Q$, hence $\mathscr{L}$ has an expression (5.3). It is easy to verify the Zermelo conditions (4.2). Indeed, applying the Lepage form criterion (5.5), we have

$$
\frac{\partial \mathscr{L}}{\partial y_{j}^{K}} y_{k}^{K}=\frac{1}{(n-1) !} A_{K_{1} K_{2} \ldots K_{n}} y_{k}^{K_{1}} y_{j_{2}}^{K_{2}} \ldots y_{j_{n}}^{K_{n}} \varepsilon^{j j_{2} \ldots j_{n}}
$$


Since $A_{K_{1} K_{2} \ldots K_{n}}$ (resp. $\varepsilon^{j j_{2} \ldots j_{n}}$ ) are skew-symmetric in subscripts (resp. superscripts), we see that the right-hand side of (5.7) vanishes whenever $j \neq k$. From (5.7) and (5.3) it follows that

$$
\frac{\partial \mathscr{L}}{\partial y_{j}^{K}} y_{j}^{K}=\frac{1}{(n-1) !} A_{K_{1} K_{2} \ldots K_{n}} y_{j}^{K_{1}} y_{j_{2}}^{K_{2}} \ldots y_{j_{n}}^{K_{n}} \varepsilon^{j j_{2} \ldots j_{n}}=n \mathscr{L}
$$

as required.

Applying Theorem 19] and Corollary 20, we obtain a mapping $\rho \rightarrow W_{h \rho}$, assigning to any Lepage form $\rho$ on $\operatorname{Imm} T_{n}^{1} Q$ the Lepage equivalent $W_{h \rho}$ of $h \rho$ defined on $G_{n}^{1} Q$ by formula (4.6).

Theorem 21. (a) For any Lagrangian $\lambda \in \Omega_{n, \mathbf{R}^{n}}^{1}\left(\mathbf{R}^{n} \times Q\right)$, $W_{\lambda}$ is a Lepage form.

(b) If $\rho$ is a Lepage form on $\operatorname{Imm} T_{n}^{1} Q$, then the fundamental Lepage equivalent $W_{h \rho}$ of $h \rho$ satisfies

$$
\left(T_{n}^{1} \zeta\right)^{*} W_{h \rho}=\left(T_{n}^{1} \zeta\right)^{*} \rho
$$

for arbitrary immersion $\zeta: U \rightarrow Q$.

(c) Let $\rho$ be a Lepage form on $\operatorname{Imm} T_{n}^{1} Q$, expressed by (5.1). Then $\rho$ coincides with the fundamental Lepage equivalent $W_{h \rho}$ of $h \rho$ if and only if the components of $\rho$ satisfy

$$
\frac{\partial^{k} A_{K_{1} \ldots K_{n-k} L_{n-k+1} \ldots L_{n}}}{\partial y_{j_{n-k+1}}^{K_{n-k+1}} \ldots \partial y_{j_{n}}^{K_{n}}} y_{j_{n-k+1}}^{L_{n-k+1}} \ldots y_{j_{n}}^{L_{n}}=0
$$

for every $k, 1 \leq k \leq n-1$.

Proof. 1. Assertion (a) is a direct consequence of the definition of $W_{\lambda}$. Indeed, one can also verify the Lepage form condition (5.5) directly for

$$
A_{K_{1} K_{2} \ldots K_{n}}=\frac{1}{n !} \frac{\partial^{n} \mathscr{L}}{\partial y_{j_{1}}^{K_{1}} \partial y_{j_{2}}^{K_{2}} \ldots \partial y_{j_{n}}^{K_{n}}} \varepsilon_{j_{1} j_{2} \ldots j_{n}}
$$

2. Since both $\rho$ and $W_{h \rho}$ are Lepage equivalents of the Lagrangian $h \rho$, the assertion (b) is implied by the definition of Lepage equivalent of a Lagrangian.

However, we prove (b) directly since a proof of assertion (c) is included. Suppose $h \rho=\mathscr{L} \omega_{0}$, where $\mathscr{L}: \operatorname{Imm} T_{n}^{1} Q \rightarrow \mathbf{R}$ is the Lagrange function expressed by (5.3). Applying the fact that $\rho$ is a Lepage form satisfying (5.5), we obtain for any $1 \leq p \leq n$,

$$
\begin{gathered}
\frac{\partial^{p} \mathscr{L}}{\partial y_{j_{1}}^{K_{1}} \ldots \partial y_{j_{p}}^{K_{p}}}=\sum_{k=0}^{p-1} \frac{1}{(n-p+k) !}\left(\begin{array}{c}
p-1 \\
k
\end{array}\right) \frac{\partial^{k} A_{K_{1} \ldots K_{p-k} L_{p-k+1} \ldots L_{n}}}{\partial y_{j_{p-k+1}}^{K_{p-k+1}} \ldots \partial y_{j_{p}}^{K_{p}}} \\
\cdot y_{s_{p-k+1}}^{L_{p-k+1}} \ldots y_{s_{n}}^{L_{n}} \varepsilon^{j_{1} \ldots j_{p-k} s_{p-k+1} \ldots s_{n}} .
\end{gathered}
$$


Hence $W_{h \rho}$, given by (4.6), is expressed as

$$
\begin{aligned}
W_{h \rho}= & \frac{1}{(n !)^{2}} \frac{\partial^{n} \mathscr{L}}{\partial y_{j_{1}}^{K_{1}} \partial y_{j_{2}}^{K_{2}} \ldots \partial y_{j_{n}}^{K_{n}}} \varepsilon_{j_{1} j_{2} \ldots j_{n}} d y^{K_{1}} \wedge d y^{K_{2}} \wedge \ldots \wedge d y^{K_{n}} \\
= & \frac{1}{(n !)^{2}}\left(\sum_{k=0}^{n-1} \frac{1}{k !}\left(\begin{array}{c}
n-1 \\
k
\end{array}\right) \frac{\partial^{k} A_{K_{1} \ldots K_{n-k} L_{n-k+1} \ldots L_{n}}}{\partial y_{j_{n-k+1}}^{K_{n-k+1}} \ldots \partial y_{j_{n}}^{K_{n}}} y_{s_{n-k+1}}^{L_{n-k+1}} \ldots y_{s_{n}}^{L_{n}}\right) \\
& \quad \cdot \varepsilon^{j_{1} \ldots j_{n-k} s_{n-k+1} \ldots s_{n}} \varepsilon_{j_{1} j_{2} \ldots j_{n}} d y^{K_{1}} \wedge d y^{K_{2}} \wedge \ldots \wedge d y^{K_{n}} \\
= & \frac{1}{(n !)^{2}}\left(\begin{array}{l}
\sum_{k=1}^{n-1} \frac{1}{k !}\left(\begin{array}{c}
n-1 \\
k
\end{array}\right) \frac{\partial^{k} A_{K_{1} \ldots K_{n-k} L_{n-k+1} \ldots L_{n}}}{\partial y_{j_{n-k+1}}^{K_{n-k+1}} \ldots \partial y_{j_{n}}^{K_{n}}} y_{j_{n-k+1}}^{L_{n-k+1}} \ldots y_{j_{n}}^{L_{n}}(n-k) ! k ! \\
\quad
\end{array}\right. \\
& \left.\quad n ! A_{K_{1} \ldots K_{n}}\right) \cdot d y^{K_{1}} \wedge d y^{K_{2}} \wedge \ldots \wedge d y^{K_{n}} .
\end{aligned}
$$

Applying the identities (4.3) into (5.8), and using (5.8) recursively for $1 \leq p \leq n$, we derive the condition

$$
\frac{\partial^{k} A_{K_{1} \ldots K_{n-k} L_{n-k+1} \ldots L_{n}}}{\partial y_{j_{n-k+1}}^{K_{n-k+1}} \ldots \partial y_{j_{n-1}}^{K_{n-1}} \partial y_{j_{n}}^{K_{n}}} y_{i_{1}}^{K_{1}} \ldots y_{i_{n-k}}^{K_{n-k}} y_{i_{n-k+1}}^{K_{n-k+1}} \ldots y_{i_{n-1}}^{K_{n-1}} y_{j_{n-k+1}}^{L_{n-k+1}} \ldots y_{j_{n}}^{L_{n}}=0
$$

for all $k$, where $1 \leq k \leq n-1$. Now, with the help of (5.10), it is straightforward to see that the pull-back operation along $T_{n}^{1} \zeta$ onto $W_{h \rho}($ (5.9) gives

$$
T_{n}^{1} \zeta^{*} W_{h \rho}=T_{n}^{1} \zeta^{*}\left(\frac{1}{n !} A_{K_{1} \ldots K_{n}} d y^{K_{1}} \wedge d y^{K_{2}} \wedge \ldots \wedge d y^{K_{n}}\right)=T_{n}^{1} \zeta^{*} \rho
$$

3. Assertion (c) directly follows from the proof of (b), the expression (5.9) of $W_{h \rho}$.

The following theorem describes a close relation of Lepage forms with the calculus of variations, well-known from the variational theory on fibered manifolds (cf. Krupka and Saunders [16], and references therein).

Theorem 22. Suppose $\rho$ is a $\pi^{1,0}$-horizontal Lepage form on $\operatorname{Imm} T_{n}^{1} Q$, and $\mathscr{L}$ : $\operatorname{Imm} T_{n}^{1} Q \rightarrow \mathbf{R}$ is the Lagrange function associated to $\rho$. Then in any chart $(V, \psi)$ on $Q$

$$
p_{1} d \rho=E_{K}(\mathscr{L}) \omega^{K} \wedge \omega_{0}
$$

where

$$
E_{K}(\mathscr{L})=\frac{\partial \mathscr{L}}{\partial y^{K}}-d_{j} \frac{\partial \mathscr{L}}{\partial y_{j}^{K}} .
$$

Proof. Suppose $\rho$ has an expression (5.1) with respect to a chart on $Q$, with coefficients satisfying the Lepage condition (5.5). We compute the exterior derivative $d \rho$ of the Lepage form $\rho$, and its 1-contact component $p_{1} d \rho$. We have

$$
d \rho=\frac{1}{(n+1) !}\left(B_{L_{1} L_{2} \ldots L_{n+1}} d y^{L_{1}}+B_{L_{1} L_{2} \ldots L_{n+1}}^{j} d y_{j}^{L_{1}}\right) \wedge d y^{L_{2}} \wedge \ldots \wedge d y^{L_{n+1}}
$$


where

$$
\begin{aligned}
& B_{L_{1} L_{2} \ldots L_{n+1}}=\frac{\partial A_{L_{2} L_{3} \ldots L_{n+1}}}{\partial y^{L_{1}}}-\frac{\partial A_{L_{1} L_{3} L_{4} \ldots L_{n+1}}}{\partial y^{L_{2}}}-\ldots-\frac{\partial A_{L_{2} L_{3} \ldots L_{n} L_{1}}}{\partial y^{L_{n+1}}}, \\
& B_{L_{1} L_{2} \ldots L_{n+1}}^{j}=(n+1) \frac{\partial A_{L_{2} L_{3} \ldots L_{n+1}}}{\partial y_{j}^{L_{1}}} .
\end{aligned}
$$

By a direct calculation we get

$$
\begin{aligned}
p_{1} d \rho & =\frac{1}{n !} B_{K L_{1} L_{2} \ldots L_{n}} y_{i_{1}}^{L_{1}} y_{i_{2}}^{L_{2}} \ldots y_{i_{n}}^{L_{n}} \varepsilon^{i_{1} i_{2} \ldots i_{n}} \omega^{K} \wedge \omega_{0} \\
& +\frac{1}{(n+1) !} B_{K L_{1} L_{2} \ldots L_{n}}^{j} y_{i_{1}}^{L_{1}} y_{i_{2}}^{L_{2}} \ldots y_{i_{n}}^{L_{n}} \varepsilon^{i_{1} i_{2} \ldots i_{n}} \omega_{j}^{K} \wedge \omega_{0} \\
& -\frac{n}{(n+1) !} B_{L_{1} K L_{2} \ldots L_{n}}^{j} y_{j i_{1}}^{L_{1}} y_{i_{2}}^{L_{2}} \ldots y_{i_{n}}^{L_{n}} \varepsilon^{i_{1} i_{2} \ldots i_{n}} \omega^{K} \wedge \omega_{0} .
\end{aligned}
$$

But from the condition (5.5) it follows that the second summand of (5.13) vanishes hence $p_{1} d \rho=\varepsilon_{K} \omega^{K} \wedge \omega_{0}$, where

$$
\begin{aligned}
\varepsilon_{K}= & \frac{1}{n !}\left(\frac{\partial A_{L_{1} L_{2} \ldots L_{n}}}{\partial y^{K}}-\frac{\partial A_{K L_{2} L_{3} \ldots L_{n}}}{\partial y^{L_{1}}}-\ldots-\frac{\partial A_{L_{1} L_{2} \ldots L_{n-1} K}}{\partial y^{L_{n}}}\right) \\
& \cdot y_{i_{1}}^{L_{1}} y_{i_{2}}^{L_{2}} \ldots y_{i_{n}}^{L_{n}} \varepsilon^{i_{1} i_{2} \ldots i_{n}}-\frac{1}{(n-1) !} \frac{\partial A_{K L_{2} \ldots L_{n}}}{\partial y_{j}^{L_{1}}} y_{j i_{1}}^{L_{1}} y_{i_{2}}^{L_{2}} \ldots y_{i_{n}}^{L_{n}} \varepsilon^{i_{1} i_{2} \ldots i_{n}}
\end{aligned}
$$

Now, using the properties of the Lagrange function $\mathscr{L}$ associated to $\rho$, it is easy to verify that the coefficients of $p_{1} d \rho$, the functions $\varepsilon_{K}$ (5.14), coincide with $E_{K}(\mathscr{L})$ given by (5.12).

The $(n+1)$-form $E_{\rho}=p_{1} d \rho$ (5.11) is called the Euler-Lagrange form associated with a Lepage form $\rho$, and its coefficients $E_{K}(\mathscr{L})$ are the Euler-Lagrange expressions of the Lagrange function $\mathscr{L}$.

5.2. The variational integral, variations, and the first variation formula. Let $\rho$ be a $\pi^{1,0}$-horizontal Lepage form on $\operatorname{Imm} T_{n}^{1} Q$, and $h \rho=\mathscr{L} \omega_{0}$ the Lagrangian associated to $\rho$, where $\mathscr{L}: \operatorname{Imm} T_{n}^{1} Q \rightarrow \mathbf{R}$ is the Lagrange function given by (5.3). Let $\zeta: U \rightarrow Q$ be an immersion on an open subset $U$ of $\mathbf{R}^{n}$. Any compact subset $\Omega$ of $U$ associates with $\rho$ the integral

$$
\rho_{\Omega}(\zeta)=\int_{\Omega}\left(T_{n}^{1} \zeta\right)^{*} \rho=\int_{\Omega}\left(\mathscr{L} \circ T_{n}^{1} \zeta\right) \omega_{0} .
$$

Since $\mathscr{L}$ is positive homogeneous (Corollary 20), it follows from Theorem 13 that the integral (5.15) is invariant with respect to reparametrizations; this means that

$$
\rho_{\Omega_{1}}(\zeta)=\rho_{\Omega_{2}}(\zeta \circ \mu)
$$

for any orientation-preserving diffeomorphism $\mu$ of $\mathbf{R}^{n}$ and any two compact subsets $\Omega_{1}, \Omega_{2} \subset U \subset \mathbf{R}^{n}$ such that $\mu\left(\Omega_{2}\right)=\Omega_{1}$. Moreover, by Theorem 21, (b), it follows that $\rho$ and $W_{h \rho}$ (4.6) define the same integral (5.15). Thus, we have

$$
\rho_{\Omega}(\zeta)=\int_{\Omega}\left(G_{n}^{1} \zeta\right)^{*} W_{h \rho},
$$

where $G_{n}^{1} \zeta$ is the Grassmann prolongation of $\zeta$ (see (3.9)).

The real-valued function $\zeta \rightarrow \rho_{\Omega}(\zeta)$, defined on the set of immersions from an open subset $U$ of $\mathbf{R}^{n}$ into $Q$, is called the variational functional associated with $\rho$ and $\Omega$. In the standard sense, we describe the behaviour of $\rho_{\Omega}$ with respect to 
variations (deformations) of $\zeta$ or, more precisely, variations of the image $\zeta(\Omega) \subset Q$. Following the geometric foundations of the calculus of variations on fibered spaces (see Krupka [14]), variations of $\zeta$ are induced by means of a vector field.

Let $\Xi$ be a vector field on an open subset $W \subset Q$, and $\alpha_{t}^{\Xi}$ be its local oneparameter group. For every immersion $\zeta: \mathbf{R}^{n} \supset U \rightarrow Q$ such that $\zeta(U) \subset W, \alpha_{t}^{\Xi} \circ \zeta$ is a one-parameter family of immersions, which depends smoothly on parameter $t$, and it is called a variation of $\zeta$ induced by the vector field $\Xi$. Since $\Omega$ is supposed to be compact, the value of the variational integral $\rho_{\Omega}\left(\alpha_{t}^{\Xi} \circ \zeta\right)$ (5.15), (5.16), is defined for all sufficiently small $t$, and we get

$$
\rho_{\Omega}\left(\alpha_{t}^{\Xi} \circ \zeta\right)=\int_{\Omega}\left(G_{n}^{1}\left(\alpha_{t}^{\Xi} \circ \zeta\right)\right)^{*} W_{h \rho}=\int_{\Omega} G_{n}^{1} \zeta^{*} G_{n}^{1} \alpha_{t}^{\Xi *} W_{h \rho},
$$

where the identity (3.11) is applied. Appling the theorem on differentiating an integral dependent on a parameter, and with the help of the concept of a Lie derivative of a differential form, we obtain

$$
\left(\frac{d}{d t} \rho_{\Omega}\left(\alpha_{t}^{\Xi} \circ \zeta\right)\right)_{0}=\int_{\Omega} G_{n}^{1} \zeta^{*}\left(\frac{d}{d t} G_{n}^{1} \alpha_{t}^{\Xi *} W_{h \rho}\right)_{0}=\int_{\Omega} G_{n}^{1} \zeta^{*} \partial_{G_{n}^{1} \Xi} W_{h \rho} .
$$

With the notation given by (5.16), we may denote this number as

$$
\left(\partial_{G_{n}^{1} \Xi} W_{h \rho}\right)_{\Omega}(\zeta)=\int_{\Omega} G_{n}^{1} \zeta^{*} \partial_{G_{n}^{1} \Xi} W_{h \rho} .
$$

(5.17) is called the variation of the variational functional $\zeta \rightarrow \rho_{\Omega}(\zeta)$ (5.16) at a point $\zeta$, induced by the vector field $\Xi$. The corresponding functional $\zeta \rightarrow\left(\partial_{G_{n}^{1} \Xi} W_{h \rho}\right)_{\Omega}(\zeta)$ is the variational derivative of $\rho_{\Omega}$ with respect to $\Xi$.

Theorem 23. Let $\rho$ be a $\pi^{1,0}$-horizontal Lepage form on $\operatorname{Imm} T_{n}^{1} Q$, and $W_{h \rho}$ be the fundamental Lepage equivalent of the Lagrangian $h$. Let $\Xi$ be a vector field on an open subset $W \subset Q$. Then the variational derivative $\partial_{G_{n}^{1} \Xi W_{h \rho}}$ of $\rho_{\Omega}$ satisfies:

(a) For every immersion $\zeta: \mathbf{R}^{n} \supset U \rightarrow Q$ such that $\zeta(U) \subset W$,

$$
G_{n}^{1} \zeta^{*} \partial_{G_{n}^{1} \Xi} W_{h \rho}=J^{2}\left(\operatorname{id}_{\mathbf{R}^{n}} \times \zeta\right)^{*} i_{J^{2} \Xi} E_{\rho}+d\left(G_{n}^{1} \zeta^{*} i_{G_{n}^{1} \Xi} W_{h \rho}\right) .
$$

(b) For every compact submanifold $\Omega$ of $U \subset \mathbf{R}^{n}$, and every immersion $\zeta: \mathbf{R}^{n} \supset$ $U \rightarrow Q$ such that $\zeta(U) \subset W$,

$$
\int_{\Omega} G_{n}^{1} \zeta^{*} \partial_{G_{n}^{1} \Xi} W_{h \rho}=\int_{\Omega} J^{2}\left(\operatorname{id}_{\mathbf{R}^{n}} \times \zeta\right)^{*} i_{J^{2} \Xi} E_{\rho}+\int_{\partial \Omega} G_{n}^{1} \zeta^{*} i_{G_{n}^{1} \Xi} W_{h \rho} .
$$

Proof. (a) Suppose $\Xi$ is a vector field on $W \subset Q$. Using the Cartan's formula, we write the Lie derivative of $W_{h \rho}$ with respect to $G_{n}^{1} \Xi$ as

$$
\partial_{G_{n}^{1} \Xi} W_{h \rho}=i_{G_{n}^{1} \Xi} d W_{h \rho}+d i_{G_{n}^{1} \Xi} W_{h \rho} .
$$

Since $W_{h \rho}$ and $\rho$ are both Lepage equivalents of the Lagrangian $h \rho$, it follows from Theorem 3 that $\left(\pi^{2,1}\right)^{*} d W_{h \rho}=E_{\rho}+F$, where $E_{\rho}$ is the Euler-Lagrange form (5.11), and $F$ has the order of contactness $\geq 2$. For any form $\eta$ defined on $G_{n}^{1} Q$, we have $\left(\pi^{2,1}\right)^{*} i_{G_{n}^{1} \Xi} \eta=i_{J^{2} \Xi}\left(\pi^{2,1}\right)^{*} \eta$, and $J^{2}\left(\operatorname{id}_{\mathbf{R}^{n}} \times \zeta\right)^{*}\left(\pi^{2,1}\right)^{*} \eta=G_{n}^{1} \zeta^{*} \eta$. Hence

$$
\left(\pi^{2,1}\right)^{*} \partial_{G_{n}^{1} \Xi} W_{h \rho}=i_{J^{2} \Xi}\left(E_{\rho}+F\right)+d\left(\left(\pi^{2,1}\right)^{*} i_{G_{n}^{1} \Xi} W_{h \rho}\right),
$$

and since the pull-back operation $J^{2}\left(\operatorname{id}_{\mathbf{R}^{n}} \times \zeta\right)^{*}$ annihilates contact forms, we get (5.18).

(b) Formula (5.19) follows from (5.18) and application of the Stokes' theorem. 
An immersion $\zeta: \mathbf{R}^{n} \supset U \rightarrow Q$ is called an extremal of the variational functional $\rho_{\Omega}$ (5.16), or (5.15), on piece $\Omega$, if for every vector field $\Xi$ such that $\Xi$ vanishes on the boundary $\partial \Omega$ of $\Omega$ along $\zeta$, and $\operatorname{supp} \Xi \cap \operatorname{Im} \zeta \subset \zeta(\Omega)$, the variational derivative of $\rho_{\Omega}$ satisfies

$$
\left(\partial_{G_{n}^{1} \Xi} W_{h \rho}\right)_{\Omega}(\zeta)=0 .
$$

This condition means that the values of the variational functional $\rho_{\Omega}$ remain stable with respect to compact deformations of the immersed submanifold $\operatorname{Im} \zeta$. The following theorem gives necessary and sufficient conditions for $\zeta$ to be an extremal of the variational functional $\rho_{\Omega}$ in terms of the Euler-Lagrange form $E_{\rho}$ (5.11).

Theorem 24. Let $\rho$ be a $\pi^{1,0}$-horizontal Lepage form on $\operatorname{Imm} T_{n}^{1} Q$, and $\zeta: \mathbf{R}^{n} \supset$ $U \rightarrow Q$ be an immersion. The following conditions are equivalent:

(a) $\zeta$ is an extremal of $\rho_{\Omega}$.

(b) The Euler-Lagrange form $E_{\rho}$ satisfies

$$
J^{2}\left(\operatorname{id}_{\mathbf{R}^{n}} \times \zeta\right)^{*} E_{\rho}=0 .
$$

(c) In any chart $(V, \psi)$ on $Q$, the following system for $\zeta$ is satisfied

$$
E_{K}(\mathscr{L}) \circ T_{n}^{2} \zeta=0, \quad 1 \leq K \leq m+n,
$$

where $h \rho=\mathscr{L} \omega_{0}$, and the left-hand side of (5.21) is given by (5.12).

Proof. We omit the proof since it follows from Theorem 23, (a), and it is a standard result of the global variational theory on fibered manifolds.

The system of second-order partial differential equations (5.21) for $\zeta$ is called the Euler-Lagrange equations, associated with the Lepage form $\rho$.

5.3. Invariant Lepage forms on $G_{n}^{1} Q$ and the Noether's theorem. Let $W$ be an open subset of $Q$, and $\tilde{W}^{1}=\left(\tau_{n, G}^{1,0}\right)^{-1}(W) \subset G_{n}^{1} Q$. Let $\eta$ be an $n$-form defined on $\tilde{W}^{1}$, and let $\alpha: W \rightarrow Q$ be a diffeomorphism. We say that $\eta$ is invariant with respect to $\alpha$, if

$$
\left(G_{n}^{1} \alpha\right)^{*} \eta=\eta \bmod \tilde{\Theta}^{1} W,
$$

where $G_{n}^{1} \alpha$ is the Grassmann prolongation of $\alpha$ (see (3.10) $)$, and $\tilde{\Theta}^{1} W$ is the contact ideal (Sec. 3). If condition (5.22) is satisfied, $\alpha$ is also said to be an invariance transformation of $\eta$. It is straightforward to apply this definition to vector fields by means of their one-parameter groups. We say that a vector field $\Xi$ on $W$ is a generator of invariance transformations of the form $\eta$, if the one-parameter group $\alpha_{t}^{\Xi}$ of $\Xi$ consists of invariance transformations of $\eta$.

Denote by $\alpha^{\Xi}$ the global flow of a vector field $\Xi$. We have the following basic formula applied to the Grassmann prolongation of a vector field.

Lemma 25. Let $\eta$ be an $n$-form on $\tilde{W}^{1} \subset G_{n}^{1} Q$, and $\Xi$ be a vector field on $W$. Then

$$
\frac{d}{d t}\left(G_{n}^{1} \alpha_{t}^{\Xi}\right)^{*} \eta\left(\left[J_{0}^{1} \zeta\right]\right)=\left(G_{n}^{1} \alpha_{t}^{\Xi}\right)^{*} \partial_{G_{n}^{1} \Xi \eta\left(\left[J_{0}^{1} \zeta\right]\right),}
$$

for every point $\left(t,\left[J_{0}^{1} \zeta\right]\right)$ of the domain of the flow $G_{n}^{1} \alpha^{\Xi}$.

Proof. Formula (5.23) is obtained by a straightforward differentiating of the curve $t \rightarrow\left(G_{n}^{1} \alpha_{t}^{\Xi}\right)^{*} \eta\left(\left[J_{0}^{1} \zeta\right]\right)$ at an arbitrary point $\left(t,\left[J_{0}^{1} \zeta\right]\right)$ of the domain of $G_{n}^{1} \alpha^{\Xi}$. 
The following result extends the classical Noether's equation to the Grassmann fibrations.

Theorem 26. Let $\eta$ be an $n$-form on $\tilde{W}^{1} \subset G_{n}^{1} Q$, and $\Xi$ be a vector field on $W$. The following two conditions are equivalent:

(a) $\Xi$ is the generator of invariance transformations of $\eta$.

(b) The Lie derivative of $\eta$ with respect to vector field $G_{n}^{1} \Xi$ satisfies

$$
\partial_{G_{n}^{1} \Xi \eta=0} \bmod \tilde{\Theta}^{1} W .
$$

Proof. The proof is a straightforward extension of an analogous result; see Urban and Krupka 22.

Corollary 27. Let $\rho$ be a $\pi^{1,0}$-horizontal Lepage form on $\operatorname{Imm} T_{n}^{1} Q$, and $W_{h \rho}$ be the fundamental Lepage equivalent of the Lagrangian $h \rho$. Let $\Xi$ be the generator of invariance transformations of $W_{h \rho}$. Then

$$
i_{J_{n}^{2} \Xi} E_{\rho}+d\left(\left(\pi^{2,1}\right)^{*} i_{G_{n}^{1} \Xi} W_{h \rho}\right)=0 \quad \bmod \Theta^{2} W,
$$

where $\Theta^{2} W$ is the ideal of forms, locally generated by contact 1 -forms $\omega^{K}=d y^{K}-$ $y_{l}^{K} d x^{l}$, and $\omega_{j}^{K}=d y_{j}^{K}-y_{j l}^{K} d x^{l}$, on a chart neighborhood of $J^{2}\left(\mathbf{R}^{n} \times Q\right)$.

Proof. Using Theorem 26. formula (5.24) is a restatement of the first variation formula (5.20) for invariant Lepage forms.

Let $\zeta: \mathbf{R}^{n} \supset U \rightarrow W \subset Q$ be an immersion. A real-valued function $\phi: G_{n}^{1} Q \supset$ $\tilde{W}^{1} \rightarrow \mathbf{R}$ is called a level set function for the immersion $\zeta$, if $d\left(\phi \circ G_{n}^{1} \zeta\right)=0$. Now we follow the geometric ideas of the theory of higher-order variational functionals on fibered manifolds (see Krupka [13]), and we extend the classical Noether's theorem to Grassmann fibrations.

Theorem 28. Let $\rho$ be a $\pi^{1,0}$-horizontal Lepage form on $\operatorname{Imm} T_{n}^{1} Q$, and $W_{h \rho}$ be the fundamental Lepage equivalent of the Lagrangian $h \rho$. Suppose an immersion $\zeta: \mathbf{R}^{n} \supset U \rightarrow Q$ be an extremal of the variational functional $\rho_{\Omega}$ (5.16). Then for every generator $\Xi$ of invariance transformations of $W_{h \rho}$,

$$
d\left(G_{n}^{1} \zeta^{*} i_{G_{n}^{1} \Xi} W_{h \rho}\right)=0 .
$$

Proof. (5.25) follows from the first variation formula (5.24) for invariant Lepage forms.

\section{EXAMPle: VARIATIONAL FUNCTIONAL FOR MINIMAL SUBMANIFOLDS}

Suppose $Q$ is a smooth manifold of dimension $n+m$, endowed with a Riemannian metric $g$, and let $\zeta: X \rightarrow Q$ be an immersion defined on an $n$-dimensional smooth manifold $X$. Let $(U, \varphi), \varphi=\left(x^{j}\right)$, be a chart on $X$, and $(V, \psi), \psi=\left(y^{K}\right)$, be a chart on $Q$ such that $\zeta(U) \subset V$. If $g$ has the expression $g=g_{K L} d y^{K} \otimes d y^{L}$, then the induced Riemannian metric $\zeta^{*} g$ on $X$ is expressed by the formula

$$
\zeta^{*} g=\left(g_{K L} \circ \zeta\right) \frac{\partial\left(y^{K} \circ \zeta\right)}{\partial x^{j}} \frac{\partial\left(y^{L} \circ \zeta\right)}{\partial x^{k}} d x^{j} \otimes d x^{k},
$$

and the induced Riemannian volume element on $X$ reads

$$
\omega_{\zeta, g}=\sqrt{\operatorname{det}\left(\left(g_{K L} \circ \zeta\right) \frac{\partial\left(y^{K} \circ \zeta\right)}{\partial x^{j}} \frac{\partial\left(y^{L} \circ \zeta\right)}{\partial x^{k}}\right)} d x^{1} \wedge d x^{2} \wedge \ldots \wedge d x^{n} .
$$


Consider now $X=\mathbf{R}^{n}$ with the global canonical coordinates $x^{j}, 1 \leq j \leq n$. The Lagrangian for the problem of $n$-dimensional minimal submanifolds $\lambda=\mathscr{L} \omega_{0}$, is defined by the positive-homogeneous function $\mathscr{L}: \operatorname{Imm} T_{n}^{1} Q \rightarrow \mathbf{R}$,

$$
\mathscr{L}\left(y^{K}, y_{j}^{K}\right)=\sqrt{\operatorname{det}\left(g_{K L} y_{j}^{K} y_{k}^{L}\right)} .
$$

Assigning to $\lambda$ the fundamental Lepage equivalent $W_{\lambda}$ (4.6), we have the corresponding variational functional (see (5.16)) for immersions $\zeta$ of $\mathbf{R}^{n}$ into $Q$, associated with a compact subset $\Omega$ of $\mathbf{R}^{n}$,

$$
\zeta \rightarrow \rho_{\Omega}(\zeta)=\int_{\Omega}\left(G_{n}^{1} \zeta\right)^{*} W_{\lambda}
$$

In [14], Krupka proposed the $n$-form $\omega$, given in a chart $(V, \psi)$ by

$$
\omega_{\lambda}=\frac{1}{n !} \frac{1}{\mathscr{L}} g_{K_{1} L_{1}} g_{K_{2} L_{2}} \ldots g_{K_{n} L_{n}} D^{L_{1} L_{2} \ldots L_{n}} d y^{K_{1}} \wedge d y^{K_{2}} \wedge \ldots \wedge d y^{K_{n}}
$$

where $D^{L_{1} L_{2} \ldots L_{n}}=\operatorname{det}\left(y_{k}^{L_{j}}\right), 1 \leq j, k \leq n$, which can be regarded as a Lagrangian for the problem of minimal submanifolds, and satisfies the condition $T_{n}^{1} \zeta^{*} \omega_{\lambda}=\omega_{\zeta, g}$. Note that $\omega_{\lambda}$ is a Lepage form on $\operatorname{Imm} T_{n}^{1} Q$, as introduced in Section 5.1 .

Theorem 29. Let $\lambda \in \Omega_{n, \mathbf{R}^{n}}^{1}\left(\mathbf{R}^{n} \times Q\right), \lambda=\mathscr{L} \omega_{0}$, be the Lagrangian of order 1 , given by (6.1). Then the Lepage equivalents of $\lambda, W_{\lambda}$ (4.6), $\Lambda_{\lambda}$ (4.9), and $\omega_{\lambda}$ (6.3) coincide, and are defined on the Grassmann fibration $G_{n}^{1} Q$.

Proof. 1. We show that $\Lambda_{\lambda}=\omega_{\lambda}$. Write

$$
\mathscr{L}^{2}=\operatorname{det}\left(g_{K L} y_{j}^{K} y_{k}^{L}\right)=g_{Q_{1} L_{1}} g_{Q_{2} L_{2}} \ldots g_{Q_{n} L_{n}} y_{1}^{Q_{1}} y_{2}^{Q_{2}} \ldots y_{n}^{Q_{n}} D^{L_{1} L_{2} \ldots L_{n}},
$$

and for any $p, 1 \leq p \leq n$, we get

$$
\begin{gathered}
\frac{\partial \mathscr{L}}{\partial y_{p}^{K}}=\frac{1}{\mathscr{L}} g_{Q_{1} L_{1}} \ldots g_{Q_{p-1} L_{p-1}} g_{K L_{p}} g_{Q_{p+1} L_{p+1}} \ldots g_{Q_{n} L_{n}} \\
\cdot y_{1}^{Q_{1}} \ldots y_{p-1}^{Q_{p-1}} y_{p+1}^{Q_{p+1}} \ldots y_{n}^{Q_{n}} D^{L_{1} L_{2} \ldots L_{n}} .
\end{gathered}
$$

A straightforward application of (6.5) now implies the expression

$$
\begin{aligned}
\frac{\partial \mathscr{L}}{\partial y_{1}^{K}} & \frac{\partial \mathscr{L}}{\partial y_{2}^{K}} \ldots \frac{\partial \mathscr{L}}{\partial y_{n}^{K n}} \\
\quad & \frac{1}{\mathscr{L}^{n}} g_{K_{1} L_{1}^{1}} g_{K_{2} L_{2}^{2}} \ldots g_{K_{n} L_{n}^{n}} D^{L_{1}^{1} L_{2}^{1} \ldots L_{n}^{1}} D^{L_{1}^{2} L_{2}^{2} \ldots L_{n}^{2}} \ldots D^{L_{1}^{n} L_{2}^{n} \ldots L_{n}^{n}} \\
& \cdot g_{Q_{2}^{1} L_{2}^{1}} \ldots g_{Q_{n}^{1} L_{n}^{1}} g_{Q_{1}^{2} L_{1}^{2}} g_{Q_{3}^{2} L_{3}^{2}} \ldots g_{Q_{n}^{2} L_{n}^{2}} \ldots \ldots g_{Q_{1}^{n} L_{1}^{n}} g_{Q_{2}^{n} L_{2}^{n}} \ldots g_{Q_{n-1}^{n} L_{n-1}^{n}} \\
& \cdot y_{2}^{Q_{2}^{1}} \ldots y_{n}^{Q_{n}^{1}} y_{1}^{Q_{1}^{2}} y_{3}^{Q_{3}^{2}} \ldots y_{n}^{Q_{n}^{2}} \ldots \ldots y_{1}^{Q_{1}^{n}} y_{2}^{Q_{2}^{n}} \ldots y_{n-1}^{Q_{n-1}^{n}} .
\end{aligned}
$$

However, from the symmetry and skew-symmetry properties of the expression (6.6), we are allowed to replace the term $D^{L_{1}^{1} L_{2}^{1} \ldots L_{n}^{1}} D^{L_{1}^{2} L_{2}^{2} \ldots L_{n}^{2}} \ldots D^{L_{1}^{n} L_{2}^{n} \ldots L_{n}^{n}}$ by the product of determinants

$$
\frac{1}{n !} \prod_{\tau} D^{L_{1}^{\tau(1)} L_{2}^{\tau(2)} \cdots L_{n}^{\tau(n)}}
$$


where $\tau$ runs through the cyclic permutations $(1,2, \ldots, n),(n, 1,2, \ldots, n-1), \ldots$, $(2,3, \ldots, n, 1)$. Using (6.4), (6.7), we now get

$$
\begin{aligned}
\Lambda_{\lambda}= & \frac{1}{\mathscr{L}^{n-1}} \frac{\partial \mathscr{L}}{\partial y_{1}^{K}} \frac{\partial \mathscr{L}}{\partial y_{2}^{K}} \ldots \frac{\partial \mathscr{L}}{\partial y_{n}^{K}} d y^{K_{1}} \wedge d y^{K_{2}} \wedge \ldots \wedge d y^{K_{n}} \\
= & \frac{1}{n} \frac{1}{\mathscr{L}^{2 n-1}} g_{K_{1} L_{1}^{1}} g_{K_{2} L_{2}^{2}} \ldots g_{K_{n} L_{n}^{n}} D^{L_{1}^{1} L_{2}^{2} \ldots L_{n}^{n}} \\
& \cdot\left(g_{Q_{1}^{n} L_{1}^{n}} g_{Q_{2}^{1} L_{2}^{1}} g_{Q_{3}^{2} L_{3}^{2}} \ldots g_{Q_{n}^{n-1} L_{n}^{n-1}} y_{1}^{Q_{1}^{n}} y_{2}^{Q_{2}^{1}} \ldots y_{n}^{Q_{n}^{n-1}} D^{L_{1}^{n} L_{2}^{1} L_{3}^{2} \ldots L_{n}^{n-1}}\right) \\
& \ldots \cdot\left(g_{Q_{1}^{2} L_{1}^{2}} g_{Q_{2}^{3} L_{2}^{3}} \ldots g_{Q_{n-1}^{n} L_{n-1}^{n}} g_{Q_{n}^{1} L_{n}^{1}} y_{1}^{Q_{1}^{n}} y_{2}^{Q_{2}^{1}} \ldots y_{n}^{Q_{n}^{n-1}} D^{L_{1}^{2} L_{2}^{3} \ldots L_{n-1}^{n} L_{n}^{1}}\right) \\
& d y^{K_{1}} \wedge d y^{K_{2}} \wedge \ldots \wedge d y^{K_{n}} \\
= & \frac{1}{n !} \frac{\left(\mathscr{L}^{2}\right)^{n-1}}{\mathscr{L}^{2 n-1}} g_{K_{1} L_{1}^{1}} g_{K_{2} L_{2}^{2}} \ldots g_{K_{n} L_{n}^{n}} D^{L_{1}^{1} L_{2}^{2} \ldots L_{n}^{n}} d y^{K_{1}} \wedge d y^{K_{2}} \wedge \ldots \wedge d y^{K_{n}} \\
= & \omega_{\lambda} .
\end{aligned}
$$

2. To show that $W_{\lambda}=\omega_{\lambda}$, we proceed by a straightforward differentiating of $\mathscr{L}$ with respect to the variables $y_{j}^{K_{j}}, 1 \leq j \leq n$, with the help of (6.5), (6.7), and the formula

$$
\frac{\partial D^{L_{1} L_{2} \ldots L_{n}}}{\partial y_{p}^{M}}=\delta_{1}^{p}\left|\begin{array}{ccc}
\delta_{M}^{L_{1}} & \delta_{M}^{L_{2}} & \ldots \delta_{M}^{L_{n}} \\
y_{2}^{L_{1}} & y_{2}^{L_{2}} & \ldots y_{2}^{L_{n}} \\
\vdots & \vdots & \vdots \\
y_{n}^{L_{1}} & y_{n}^{L_{2}} & \ldots y_{n}^{L_{n}}
\end{array}\right|+\ldots+\delta_{n}^{p}\left|\begin{array}{ccc}
y_{1}^{L_{1}} & y_{1}^{L_{2}} & \ldots y_{1}^{L_{n}} \\
y_{2}^{L_{1}} & y_{2}^{L_{2}} & \ldots y_{2}^{L_{n}} \\
\vdots & \vdots & \vdots \\
\delta_{M}^{L_{1}} & \delta_{M}^{L_{2}} & \ldots \delta_{M}^{L_{n}}
\end{array}\right|
$$

Hence we obtain

as required.

$$
\frac{\partial^{n} \mathscr{L}}{\partial y_{1}^{K_{1}} \partial y_{2}^{K_{2}} \ldots \partial y_{n}^{K_{n}}}=\frac{1}{\mathscr{L}} g_{K_{1} L_{1}} g_{K_{2} L_{2} \ldots} g_{K_{n} L_{n}} D^{L_{1} L_{2} \ldots L_{n}}
$$

Remark 30. A relationship between the Hilbert-Carathéodory form $\Lambda_{\lambda}$ and the fundamental Lepage equivalent $W_{\lambda}$ of the corresponding positive-homogeneous Lagrangian $\lambda$ was also studied by Crampin and Saunders [5] for trivial Lagrangians; it is proved that a Lagrange function of the form $\mathscr{L}=\operatorname{det}\left(d_{j} f^{k}\right)$, where $f^{k}$, $1 \leq k \leq n$, are some functions on $Q$, has the property that the forms $\Lambda_{\lambda}$ and $W_{\lambda}$ coincide. Theorem 29] shows, however, a different example of a positive-homogeneous Lagrangian with this property, the minimal submanifolds Lagrangian.

By Theorem 24, (5.21), the equations for extremals, associated with the Lagrange function $\mathscr{L}$ (6.1), read

$$
E_{K}(\mathscr{L}) \circ T_{n}^{2} \zeta=0, \quad 1 \leq K \leq m+n,
$$

where

$$
\begin{gathered}
E_{K}(\mathscr{L})=\frac{n}{2} \frac{1}{\mathscr{L}} \frac{\partial g_{Q_{1} L_{1}}}{\partial y^{K}} g_{Q_{2} L_{2}} \ldots g_{Q_{n} L_{n}} y_{1}^{Q_{1}} y_{2}^{Q_{2}} \ldots y_{n}^{Q_{n}} D^{L_{1} L_{2} \ldots L_{n}} \\
-\sum_{j=1}^{n} d_{j}\left(\frac{1}{\mathscr{L}^{2}} g_{Q_{1} L_{1}} \ldots g_{Q_{j-1} L_{j-1}} g_{K L_{j}} g_{Q_{j+1} L_{j+1}} \ldots g_{Q_{n} L_{n}}\right. \\
\left.\cdot y_{1}^{Q_{1}} \ldots y_{j-1}^{Q_{j-1}} y_{j+1}^{Q_{j+1}} \ldots y_{n}^{Q_{n}} D^{L_{1} L_{2} \ldots L_{n}}\right) .
\end{gathered}
$$


(6.8) is the minimal submanifolds equation.

We conclude this section with an application of the Noether's theorem (Theorem 28) to the problem of 2-dimensional immersed minimal submanifolds of a Euclidean space.

Consider the Euclidean space $Q=\mathbf{R}^{m+2}$ with its canonical smooth manifold structure. Let $\Xi$ be a vector field on $\mathbf{R}^{m+2}$, and $G_{2}^{1} \Xi$ be its first-order Grassmann prolongation, expressed in the $(i)=\left(i_{1}, i_{2}\right)$-subordinate $\operatorname{chart}\left(\tilde{V}_{n}^{1(i)}, \tilde{\chi}_{n}^{1(i)}\right), \tilde{\chi}_{n}^{1(i)}=$ $\left(w^{i_{1}}, w^{i_{2}}, w^{\mu}, w_{i_{1}}^{\mu}, w_{i_{2}}^{\mu}\right)$, on $G_{2}^{1} \mathbf{R}^{m+2}$ by

$$
\begin{aligned}
\Xi & =\Xi^{i_{1}} \frac{\partial}{\partial w^{i_{1}}}+\Xi^{i_{2}} \frac{\partial}{\partial w^{i_{2}}}+\Xi^{\mu} \frac{\partial}{\partial w^{\mu}}, \\
G_{2}^{1} \Xi & =\Xi^{i_{1}} \frac{\partial}{\partial w^{i_{1}}}+\Xi^{i_{2}} \frac{\partial}{\partial w^{i_{2}}}+\Xi^{\mu} \frac{\partial}{\partial w^{\mu}}+\Xi_{i_{1}}^{\mu} \frac{\partial}{\partial w_{i_{1}}^{\mu}}+\Xi_{i_{2}}^{\mu} \frac{\partial}{\partial w_{i_{2}}^{\mu}},
\end{aligned}
$$

where $\Xi_{p}^{\mu}, p=i_{1}, i_{2}$, has the expression (cf. Lemma (11))

$$
\Xi_{p}^{\mu}=\frac{\partial \Xi^{\mu}}{\partial w^{p}}+w_{p}^{\nu} \frac{\partial \Xi^{\mu}}{\partial w^{\nu}}-w_{i_{1}}^{\mu} \frac{\partial \Xi^{i_{1}}}{\partial w^{p}}-w_{i_{2}}^{\mu} \frac{\partial \Xi^{i_{2}}}{\partial w^{p}}-w_{i_{1}}^{\mu} w_{p}^{\nu} \frac{\partial \Xi^{i_{1}}}{\partial w^{\nu}}-w_{i_{2}}^{\mu} w_{p}^{\nu} \frac{\partial \Xi^{i_{2}}}{\partial w^{\nu}}
$$

(no summation through $i_{1}, i_{2}$ ). A straightforward computation of the Lie derivative of the Lepage equivalent $W_{\lambda}=\omega_{\lambda}$ with respect to $G_{2}^{1} \Xi$ leads to the Noether's equation $\partial_{G_{2}^{1} \Xi} \omega_{\lambda}=0 \bmod \tilde{\Theta}^{1}$ for unknowns $\Xi$, which has the form

$$
\partial_{G_{2}^{1} \Xi} \tilde{\mathscr{L}}_{G}+\tilde{\mathscr{L}}_{G}\left(\frac{\partial \Xi^{i_{1}}}{\partial w^{i_{1}}}+\frac{\partial \Xi^{i_{1}}}{\partial w^{\nu}} w_{i_{1}}^{\nu}+\frac{\partial \Xi^{i_{2}}}{\partial w^{i_{2}}}+\frac{\partial \Xi^{i_{2}}}{\partial w^{\nu}} w_{i_{2}}^{\nu}\right)=0
$$

where $\partial_{G_{2}^{1} \Xi} \tilde{\mathscr{L}}_{G}$ denotes the Lie derivative of function $\tilde{\mathscr{L}}_{G}: G_{2}^{1} \mathbf{R}^{m+2} \rightarrow \mathbf{R}$ with respect to $G_{2}^{1} \Xi$, and $\tilde{\mathscr{L}}_{G}$ is uniquely defined Grassmann projection of the Lagrange function $\mathscr{L}$ (6.1), $\mathscr{L}=\left(w_{1}^{i_{1}} w_{2}^{i_{2}}-w_{1}^{i_{2}} w_{2}^{i_{1}}\right) \cdot \tilde{\mathscr{L}}_{G}$ (see (4.4) $)$.

Moreover, if $Q=\mathbf{R}^{m+2}$ is considered with the Euclidean metric $g=\left(\delta_{K L}\right)$, it is easy to show that a vector field $\xi$ with constant coefficients is a general solution of equation (6.9). Hence, we obtain $(m+2)$-dimensional Lie algebra of generators of invariance transformations of the Lepage equivalent $\omega_{\lambda}$, generated by the vector fields $\xi_{1}=\partial / \partial w^{i_{1}}, \xi_{2}=\partial / \partial w^{i_{2}}, \xi_{\mu}=\partial / \partial w^{\mu}$. Contracting the Lepage form $\omega_{\lambda}$ by the generators of invariance transformations, we obtain the Noether currents (first integrals), locally expressed by

$$
i_{\xi_{1}} \omega_{\lambda}=\frac{1}{\tilde{\mathscr{L}}_{G}}\left(\sum_{\mu} w_{i_{2}}^{\mu} d w^{\mu}+d w^{i_{2}}\right), \quad i_{\xi_{2}} \omega_{\lambda}=-\frac{1}{\tilde{\mathscr{L}}_{G}}\left(\sum_{\mu} w_{i_{1}}^{\mu} d w^{\mu}+d w^{i_{1}}\right),
$$

$$
i_{\xi_{\mu}} \omega_{\lambda}=\frac{1}{\tilde{\mathscr{L}}_{G}}\left(\sum_{\sigma}\left(w_{i_{1}}^{\mu} w_{i_{2}}^{\sigma}-w_{i_{1}}^{\sigma} w_{i_{2}}^{\mu}\right) d w^{\sigma}-w_{i_{2}}^{\mu} d w^{i_{1}}+w_{i_{1}}^{\mu} d w^{i_{2}}\right),
$$

where

$$
\tilde{\mathscr{L}}_{G}=\sqrt{\sum_{\sigma_{1}<\sigma_{2}}\left(w_{i_{1}}^{\sigma_{1}} w_{i_{2}}^{\sigma_{2}}-w_{i_{1}}^{\sigma_{2}} w_{i_{2}}^{\sigma_{1}}\right)^{2}+\sum_{\sigma}\left(\left(w_{i_{1}}^{\sigma}\right)^{2}+\left(w_{i_{2}}^{\sigma}\right)^{2}\right)+1} .
$$

By Theorem 28, linear forms (6.10) are closed hence also exact along every extremal $\zeta: \mathbf{R}^{2} \supset U \rightarrow \mathbf{R}^{m+2}$ of the variational functional (6.2). This result extends the concept of a Noether current as a level-set function for extremals, known in the classical mechanics (cf. Urban and Krupka [22, 23]). 
Finally, we consider 2-dimensional nonparametric minimal surfaces of $\mathbf{R}^{3}$. Write $y^{1}=x, y^{2}=y, y^{3}=z$, the canonical global coordinates of $\mathbf{R}^{3}$, and $y_{j}^{1}=x_{j}, y_{j}^{2}=$ $y_{j}, y_{j}^{3}=z_{j}, j=1,2$, the associated coordinates of $T_{2}^{1} \mathbf{R}^{3}$. Consider a subordinate $\operatorname{chart}\left(\tilde{V}_{2}^{1,(i)}, \tilde{\chi}_{2}^{1,(i)}\right)$ on $G_{2}^{1} \mathbf{R}^{3}=\operatorname{Imm} T_{2}^{1} \mathbf{R}^{3} / G L_{2}(\mathbf{R})$, where, for instance, $\left(i_{1}, i_{2}\right)=$ $(1,2)$, and $\tilde{\chi}_{2}^{1,(i)}=\left(w^{1}, w^{2}, w^{3}, w_{1}^{3}, w_{2}^{3}\right)$, where $w^{1}=x, w^{2}=y, w^{3}=z$, and

$$
w_{1}^{3}=\frac{y_{2} z_{1}-y_{1} z_{2}}{x_{1} y_{2}-x_{2} y_{1}}, \quad w_{2}^{3}=\frac{x_{1} z_{2}-x_{2} z_{1}}{x_{1} y_{2}-x_{2} y_{1}}
$$

(cf. Section 3. (3.5)). It is now easy to verify that for $Q=\mathbf{R}^{3}$ with the Euclidean metric $\delta_{K L}$, the Euler-Lagrange equations (6.8) for graph of a surface $\zeta: \mathbf{R}^{2} \supset U \rightarrow$ $\mathbf{R}^{3}, \zeta(x, y)=(x, y, u(x, y))$, are equivalent to the well-known single second-order differential equation for an unknown function $u: \mathbf{R}^{2} \supset U \rightarrow \mathbf{R}$,

$$
\left(1+u_{y}^{2}\right) u_{x x}-2 u_{x} u_{y} u_{x y}+\left(1+u_{x}^{2}\right) u_{y y}=0,
$$

cf. Dierkes, Hildebrandt, and Sauvigny [7].

Contracting the Lepage form $\omega_{\lambda}$ by the generators of invariance transformations $\partial / \partial x, \partial / \partial y, \partial / \partial z$, we get the Noether currents (6.10) hence the conservation law equations of the form

$$
\begin{aligned}
& \frac{\left(x_{1} y_{2}-x_{2} y_{1}\right) d y-\left(z_{1} x_{2}-z_{2} x_{1}\right) d z}{\sqrt{\left(y_{1} z_{2}-y_{2} z_{1}\right)^{2}+\left(z_{1} x_{2}-z_{2} x_{1}\right)^{2}+\left(x_{1} y_{2}-x_{2} y_{1}\right)^{2}}}=d f(x, y), \\
& \frac{\left(y_{1} z_{2}-y_{2} z_{1}\right) d z-\left(x_{1} y_{2}-x_{2} y_{1}\right) d x}{\sqrt{\left(y_{1} z_{2}-y_{2} z_{1}\right)^{2}+\left(z_{1} x_{2}-z_{2} x_{1}\right)^{2}+\left(x_{1} y_{2}-x_{2} y_{1}\right)^{2}}}=d g(x, y), \\
& \frac{\left(z_{1} x_{2}-z_{2} x_{1}\right) d x-\left(y_{1} z_{2}-y_{2} z_{1}\right) d y}{\sqrt{\left(y_{1} z_{2}-y_{2} z_{1}\right)^{2}+\left(z_{1} x_{2}-z_{2} x_{1}\right)^{2}+\left(x_{1} y_{2}-x_{2} y_{1}\right)^{2}}}=d h(x, y),
\end{aligned}
$$

where $f, g, h$, are arbitrary functions on $U \subset \mathbf{R}^{2}$. The Noether theorem 28 says that every minimal surface $\zeta$ of $\mathbf{R}^{3}$ is a solution of (6.12). Conversely, we claim that every solution $\zeta: \mathbf{R}^{2} \supset U \rightarrow \mathbf{R}^{3}, \zeta(x, y)=(x, y, u(x, y))$, of the conservation law equations (6.12) is a minimal surface of $\mathbf{R}^{3}$ hence an extremal of the variational functional (6.2). Indeed, using the coordinate expressions of $G_{2}^{1} \zeta: \mathbf{R}^{2} \supset U \rightarrow G_{2}^{1} \mathbf{R}^{3}$ (3.9), equations (6.12) form a Pfaffian system

$$
\frac{u_{x} u_{y} d x+\left(1+\left(u_{y}\right)^{2}\right) d y}{\sqrt{\left(u_{x}\right)^{2}+\left(u_{y}\right)^{2}+1}}=d f, \quad \frac{-\left(1+\left(u_{x}\right)^{2}\right) d x-u_{x} u_{y} d y}{\sqrt{\left(u_{x}\right)^{2}+\left(u_{y}\right)^{2}+1}}=d g
$$

and

$$
\frac{-u_{y} d x+u_{x} d y}{\sqrt{\left(u_{x}\right)^{2}+\left(u_{y}\right)^{2}+1}}=d h
$$

These conditions imply that $u_{x} d f(x, y)+u_{y} d g(x, y)=d h(x, y)$ or, equivalently,

$$
u_{x} \frac{\partial f}{\partial x}+u_{y} \frac{\partial g}{\partial x}-\frac{\partial h}{\partial x}=0, \quad u_{x} \frac{\partial f}{\partial y}+u_{y} \frac{\partial g}{\partial y}-\frac{\partial h}{\partial y}=0 .
$$

Differentiating the first equation of (6.14) with respect to $y$, the latter one with respect to $x$, and subtracting we get

$$
u_{x x} \frac{\partial f}{\partial y}-u_{x y}\left(\frac{\partial f}{\partial x}-\frac{\partial g}{\partial y}\right)-u_{y y} \frac{\partial g}{\partial x}=0 .
$$


Substituting now into this equation the partial derivatives of $f$ and $g$, determined by (6.13), we conclude that $u=u(x, y)$ is a solution of the minimal surface equation (6.11).

Remark 31. Equivalence of the Euler-Lagrange equations for extremals on one side, and a system of conservation law equations on the other side, is not understood in general yet. Our results for the minimal submanifolds problem extend particular examples from geometric mechanics which illustrate this phenomena, see Urban and Krupka [22, 23].

\section{REFERENCES}

[1] D.E. Betounes, Extension of the classical Cartan form, Phys. Rev. D 29 (1984), 599-606.

[2] J. Brajerčík and D. Krupka, Variational principles for locally variational forms, J. Math. Phys. 46 (052903) (2005), 1-15.

[3] C. Carathéodory, Über die Variationsrechnung bei mehrfachen Integralen, Acta Szeged Sect. Sci. Math. 4 (1929), 193-216.

[4] M. Crampin, D.J. Saunders, The Hilbert-Carathéodory form for parametric multiple integral problems in the calculus of variations, Acta Appl. Math. 76 (2003), 37-55.

[5] M. Crampin and D. J. Saunders, On null Lagrangians, Diff. Geom. Appl. 22 (2005), 131-146.

[6] P. Dedecker, On the generalization of symplectic geometry to multiple integrals in the calculus of variations, in: K. Bleuler and A. Reetz (eds), Differential Geometrical Methods in Mathematical Physics, Lecture Notes in Mathematics, Vol. 570 (Springer, Berlin, 1977), pp. 395-456.

[7] U. Dierkes, S. Hildebrandt, and F. Sauvigny, Minimal surfaces, 2nd Edition, Grundlehren math. Wissenschaften 339, Springer-Verlag, Berlin, 2010.

[8] G. Giachetta, L. Mangiarotti, and G. Sardanashvily, Advanced classical field theory, World Scientific, Singapore, 2009.

[9] D. R. Grigore, Lagrangian formalism on Grassmann manifolds, in: D. Krupka and D. Saunders (Eds.), Handbook of Global Analysis, Elsevier, Amsterdam, 2008, pp. 325-371.

[10] D. R. Grigore and D. Krupka, Invariants of velocities and higher-order Grassmann bundles, J. Geom. Phys. 24 (1998), 244-264.

[11] Y. Kossmann-Schwarzbach, The Noether Theorems, Springer-Verlag, New York, 2011.

[12] D. Krupka, A map associated to the Lepagean forms of the calculus of variations in fibered manifolds, Czech. Math. J. 27 (1977), 114-118.

[13] D. Krupka, Lepagean forms in higher-order variational theory, in: Modern Developments in Analytical Mechanics (Academy of Sciences of Turin, Elsevier, 1983), pp. 197-238.

[14] D. Krupka, Global variational functionals on fibered spaces, Nonlinear Analysis 47 (2001) 2633-2642.

[15] D. Krupka, Lepage forms in Kawaguchi spaces and the Hilbert form, Publ. Math. Debrecen 84, 1-2 (2014) 147-164.

[16] D. Krupka, Introduction to Global Variational Geometry, Atlantis Studies in Variational Geometry, Vol. 1, Atlantis Press, Amsterdam-Beijing-Paris, 2015.

[17] D. Krupka, O. Krupková, and D. Saunders, The Cartan form and its generalizations in the calculus of variations, Int. J. Geom. Meth. Mod. Phys. 7(4) (2010) 631-654.

[18] G. Manno and R. Vitolo, Geometric aspects of higher-order variational principles on submanifolds, Acta Appl. Math. 101 (2008) 215-229.

[19] M. A. McKiernan, Sufficiency of parameter invariance conditions in areal and higher-order Kawaguchi spaces, Publ. Math. Debrecen 13 (1966) 77-85.

[20] D.J. Saunders and M. Crampin, The fundamental form of a homogeneous Lagrangian in two independent variables, J. Geom. Phys. 60, No. 11 (2010), 1681-1697.

[21] Z. Urban and D. Krupka, Variational sequences in mechanics on Grassmann fibrations, Acta Appl. Math. 112(2) (2010) 225-249.

[22] Z. Urban and D. Krupka, Foundations of higher-order variational theory on Grassmann fibrations, Int. J. Geom. Meth. Mod. Phys. 11, No. 7 (2014) 1460023.

[23] Z. Urban and D. Krupka, Variational theory on Grassmann fibrations: Examples, Acta Math. Acad. Paed. Nyíregyhasiensis 31, No. 1 (2015) 153-170. 
[24] Z. Urban and D. Krupka, The Zermelo conditions and higher order homogeneous functions, Publ. Math. Debrecen 82, No. 1 (2013) 59-76.

[25] J. Volná and Z. Urban, First-order Variational Sequences in Field Theory, in: D. Zenkov (Ed.), The Inverse Problem of the Calculus of Variations, Local and Global Theory, Atlantis Press, Amsterdam-Beijing-Paris, 2015, pp. 215-284.

Z. Urban, Department of Mathematics and Descriptive Geometry, VŠB-Technical University of Ostrava, 17. listopadu 15, 70833 Ostrava-Poruba, Czech Republic

E-mail address: zbynek.urban@vsb.cz; urbanzp@gmail.com

J. Brajerčík, Department of Physics, Mathematics and Techniques, University of Prešov, 17. novembra 1, 08116 Prešov, Slovakia

E-mail address: jan.brajercik@unipo.sk 